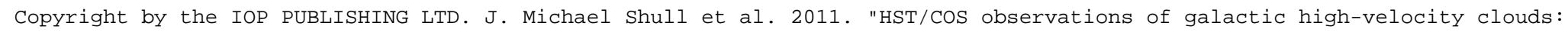
four active galactic nucleus sight lines through complex C," ApJ 739 105 doi:10.1088/0004-637x/739/2/105

\title{
HST/COS OBSERVATIONS OF GALACTIC HIGH-VELOCITY CLOUDS: FOUR ACTIVE GALACTIC NUCLEUS SIGHT LINES THROUGH COMPLEX C*
}

\author{
J. Michael Shull ${ }^{1}$, Matthew Stevans ${ }^{1}$, Charles Danforth $^{1}$, Steven V. Penton $^{1}$, Felix J. Lockman $^{2}$, And Nahum Arav $^{3}$ \\ ${ }^{1}$ CASA, Department of Astrophysical and Planetary Sciences, University of Colorado, 389-UCB, Boulder, CO 80309, USA; \\ michael.shull@colorado.edu, matthew.stevans@ colorado.edu, charles.danforth@colorado.edu, steven.penton@colorado.edu \\ ${ }^{2}$ National Radio Astronomy Observatory, Green Bank, WV 29444, USA; jlockman @ nrao.edu \\ ${ }^{3}$ Department of Physics, Virginia Tech, Blacksburg, VA 24061, USA; arav@ vt.edu \\ Received 2011 May 10; accepted 2011 July 15; published 2011 September 15
}

\begin{abstract}
We report ultraviolet spectra of Galactic high-velocity clouds (HVCs) in Complex C, taken by the Cosmic Origins Spectrograph (COS) on the Hubble Space Telescope (HST), together with new $21 \mathrm{~cm}$ spectra from the Green Bank Telescope. The wide spectral coverage and higher signal-to-noise ratio, compared to previous $H S T$ spectra, provide better velocity definition of the HVC absorption, additional ionization species (including high ions), and improved abundances in this halo gas. Complex $\mathrm{C}$ has a metallicity of $10 \%-30 \%$ solar and a wide range of ions, suggesting dynamical and thermal interactions with hot gas in the Galactic halo. Spectra in the COS medium-resolution G130M (1133-1468 $)$ and G160M (1383-1796 $)$ gratings detect ultraviolet absorption lines from eight elements in low-ionization states (O I, N I, C II, S II, Si II, Al II, Fe II, P II) and three elements in intermediate- and high-ionization states ( $\mathrm{Si}$ III, Si IV, C IV, N v). Our four active galactic nucleus sight lines toward Mrk 817, Mrk 290, Mrk 876, and PG 1259+593 have high-velocity H I and O vi column densities, $\log N_{\mathrm{HI}}=$ $19.39-20.05$ and $\log N_{\mathrm{OVI}}=13.58-14.10$, with substantial amounts of kinematically associated photoionized gas. The high-ion abundance ratios are consistent with cooling interfaces between photoionized and collisionally ionized gas: $N(\mathrm{C}$ IV $) / N(\mathrm{O}$ vI $) \approx 0.3-0.5, N(\mathrm{Si} \mathrm{IV}) / N(\mathrm{O}$ vI $) \approx 0.05-0.11, N(\mathrm{~N} \mathrm{v}) / N(\mathrm{O}$ vI $) \approx 0.07-0.13$, and $N(\mathrm{Si}$ IV $) / N(\mathrm{Si}$ III $) \approx 0.2$.
\end{abstract}

Key words: Galaxy: halo - ISM: clouds - ultraviolet: general

Online-only material: color figures

\section{INTRODUCTION}

Absorption spectra in the ultraviolet (UV) provide sensitive diagnostics of conditions in the halo of the Milky Way, where competing process of accretion and outflow determine the evolution of the Galaxy. Recent measurements (Shull et al. 2009) provide considerable insight into the infall of lowmetallicity gas onto the disk, an ongoing process that can account for the observed stellar metallicities, star formation rates, and mass-metallicity relations (Pagel 1994; Gilmore 2001; Tremonti et al. 2004). Galactic high-velocity H I clouds (HVCs) are plausible candidates for this fresh material. However, as long as they were only observed in the $21 \mathrm{~cm}$ line, their overall properties remained somewhat obscure. In recent years, sensitive UV and optical spectroscopy have revitalized this field, providing metallicities (10\%-20\% solar) and reliable distance measurements to several prominent clouds such as Complex C, Complex M, and the Magellanic Stream.

The infall of low-metallicity gas onto the Milky Way is a crucial component of most models of Galactic formation and evolution (Gibson et al. 2001) required to explain the metallicity distribution of nearby $\mathrm{G}$ and $\mathrm{K}$ dwarfs, the so-called G-dwarf problem (Pagel 1994). Infall models are attractive because Galactic disk formation is believed to occur by the gradual accretion of pristine or partially processed material into the interstellar medium (ISM). The metallicity of the initial reservoir of gas is enriched by ejecta from star formation and mixing with infalling low-metallicity gas, possibly through

\footnotetext{
* Based on observations made with the NASA/ESA Hubble Space Telescope, obtained from the data archive at the Space Telescope Science Institute. STScI is operated by the Association of Universities for Research in Astronomy, Inc., under NASA contract NAS5-26555.
}

"cold-mode accretion" (Dekel \& Birnboim 2006; Kereš et al. 2009). This process continues to the present day, regulated in a manner that produces the local G-dwarf metallicity distribution and avoids the overproduction of metal-poor disk stars. The infall of gas from the intergalactic medium (IGM) and low Galactic halo also places a chemical imprint on mass-metallicity relations (Erb et al. 2006).

One likely manifestation of infall from the halo into the disk may have been observed in the system of Galactic HVCs. These HVCs were defined as neutral hydrogen clouds moving at velocities incompatible with differential Galactic rotation (Wakker \& van Woerden 1997). First discovered in $21 \mathrm{~cm}$ emission, Galactic HVCs have become even more interesting when observed in UV absorption lines of heavy elements (Wakker et al. 1999; Gibson et al. 2000, 2001; Richter et al. 2001; Sembach et al. 1999, 2003; Collins et al. 2003, 2004, 2005, 2007, 2009; Fox et al. 2004, 2006). Hereafter, we denote the Collins et al. papers as CSG03, CSG07, CSG09, etc. The UV data also demonstrate that HVCs are more extended on the sky at lower total hydrogen column densities. Their "extended atmospheres" often contain more ionized gas than neutral gas, as seen in their $\mathrm{H} \alpha$ emission (Tufte et al. 1998).

The UV absorption-line surveys find that a greater fraction of the high-latitude sky is covered with infalling ionized gas than would have been suspected from $21 \mathrm{~cm}$ data. In $21 \mathrm{~cm}$ emission, the HVC sky-covering factor is $37 \%$ down to column densities $\mathrm{N}_{\mathrm{H} \text { I }} \gtrsim 8 \times 10^{17} \mathrm{~cm}^{-2}$ at the $4 \sigma$ level (Lockman et al. 2002). In surveys using the more sensitive UV absorption lines, the sky coverage is much higher: $\gtrsim 60 \%$ in O VI $\lambda 1031.9$ (Sembach et al. 2003) and $81 \% \pm 5 \%$ in Si III $\lambda 1206.5$ (Shull et al. 2009). Owing to its large oscillator strength, the Si III absorption line is the best probe of ionized HVCs, typically four to five 
times stronger than O vi. From our Hubble Space Telescope (HST)/Space Telescope Imaging Spectrograph (STIS) survey of high-velocity Si III (Shull et al. 2009; CSG09), we infer that the low Galactic halo is enveloped by a sheath of ionized, lowmetallicity gas, which can provide a substantial cooling inflow $\left(\sim 1 M_{\odot} \mathrm{yr}^{-1}\right)$ to help replenish star formation in the Galactic disk, estimated at 2-4 $M_{\odot} \mathrm{yr}^{-1}$ (Diehl et al. 2006; Robitaille \& Whitney 2010).

Measuring the column densities, metallicities, and ionization conditions in HVCs are key steps in elucidating their importance in Galactic evolution. These parameters are best measured in the UV, where a rich variety of elements and ion stages is accessible through their resonance absorption lines, which are sensitive to column densities well below that detectable in $21 \mathrm{~cm}$ emission. For example, Si III $\lambda 1206.5$ is easily detectable with HST at column densities $N_{\text {Si III }} \gtrsim 10^{12} \mathrm{~cm}^{-2}$, corresponding to total hydrogen column densities $\mathrm{N}_{\mathrm{H}} \gtrsim$ $\left(3 \times 10^{16} \mathrm{~cm}^{-2}\right)\left(Z_{\odot} / Z\right)$, scaling inversely with metallicity. The high-quality data achievable with the Cosmic Origins Spectrograph (COS; signal-to-noise ratio $(\mathrm{S} / \mathrm{N}) \gtrsim 30$ ) provide much better definition of the velocity extent of these HVCs, many of which do not exhibit narrow Gaussian absorption profiles. The ions commonly accessible to the COS G130/G160M gratings include O I, N I, N v, C II, C IV, Si II, Si III, Si IV, S II, Al II, Fe II, Ni II, and P II.

In this paper, we present high-quality, far-UV spectroscopic observations of absorption along four sight lines passing through Complex C. One of the most prominent HVCs, Complex C, extends over Galactic longitudes from $\ell \approx 30^{\circ}$ to $150^{\circ}$ in the northern Galactic hemisphere (see maps by Wakker 2001; CSG03; Fox et al. 2004). Multiple sight lines pierce Complex C, with UV-determined metallicities ranging from $10 \%$ to $30 \%$ solar (Wakker et al. 1999; CSG03; CSG07). The recent distance estimate of $d=10 \pm 2.5 \mathrm{kpc}$ (Wakker et al. 2007; Thom et al. 2008) confirms that it has a substantial mass $\left(\sim 10^{7} M_{\odot}\right)$. As Complex $\mathrm{C}$ falls into the Galactic disk over the next 50-100 Myr, it will deliver an average mass inflow of $\sim 0.1 M_{\odot} \mathrm{yr}^{-1}$, some $5 \%$ of the disk replenishment rate for star formation.

Our spectral data were obtained with the moderate-resolution ( $R \approx 18,000$ ) gratings, G130M and G160M, on COS on board HST (Green et al. 2011; Osterman et al. 2011). The typical COS wavelength coverage is from 1133 to $1796 \AA$, although individual spectra extend slightly outside this range: Mrk 817 (1134.5-1796.1 ̊), Mrk 876 (1135.4-1795.3 А), Mrk 290 (1134.1-1796.2 $\AA$ ), and PG 1259+593 (1133.9-1796.1 $\AA$ ). These UV spectra demonstrate COS capabilities for detecting HVCs along sight lines to background active galactic nucleus (AGN), with several improvements over previous studies. First, the much greater far-UV throughput of the COS gratings provides higher $\mathrm{S} / \mathrm{N}$ and better photometric accuracy. Second, the low background of the COS detectors allows us to characterize the zero flux levels, important for measurements of column densities of mildly saturated absorption lines. Third, the combination of G130M/G160M gratings offers broad wavelength coverage and access to numerous ion species and resonant lines not covered previously with either the Goddard High Resolution Spectrograph (GHRS) or the Far-Ultraviolet Spectroscopic Explorer (FUSE). In particular, our COS spectra measure species (Al II, C IV, Si IV) and transitions (O I, Si II, Fe II, P II) not typically measured or reported in GHRS data. Detecting HVCs in multiple ionization stages of the same element such as silicon (Si II, Si III, Si IV) provides diagnostics of the ionization conditions and metallicity of the HVCs, while higher ioniza- tion stages (C IV, N v, O vi, Si III, Si IV) are useful in separating the contribution of hot collisionally ionized gas from warm photoionized gas.

In Section 2, we discuss the observations and data-reduction techniques for the COS gratings (G130M and G160M) and $21 \mathrm{~cm}$ spectra from the $\mathrm{NRAO}^{4}$ Green Bank Telescope (GBT). In Section 3, we display the data and describe our analysis. In Section 4, we summarize our observations and their implications for Complex C (metallicity, ionization state, and velocity structure).

\section{OBSERVATIONS OF COMPLEX C}

In this section, we describe the HST/COS ultraviolet spectra of four AGN sight lines (Table 1) passing through Complex $\mathrm{C}$, with absorption typically appearing at local standard of rest (LSR) velocities $V_{\mathrm{LSR}} \approx-160$ to $-90 \mathrm{~km} \mathrm{~s}^{-1}$. Figure 1 illustrates the locations of our background AGN targets relative to the $21 \mathrm{~cm}$ emission from the Leiden-Argentine-Bonn (LAB) survey (Kalberla et al. 2005) with angular resolution $\sim 0.6$, spectral resolution $1.3 \mathrm{~km} \mathrm{~s}^{-1}$, and $70-90 \mathrm{mK}$ rms noise in brightness temperature $\left(T_{b}\right)$. These four sight lines exhibit a wide range in $\mathrm{H}_{\mathrm{I}}$ column densities from $\log N_{\mathrm{H}_{\mathrm{I}}}=19.39$ to 20.05 (Table 2). Figure 2 shows the combined G130M/ G160M ultraviolet spectra from COS, over the wavelength range 1135-1796 A. New $21 \mathrm{~cm}$ spectra taken at the GBT are shown in Figure 3.

\subsection{Hubble/COS Observations}

Our selected AGN sight lines (Mrk 290, Mrk 817, Mrk 876, PG 1259+593) are the first of many AGN targets, scheduled over three years of COS Guaranteed Time Observations (GTOs) that probe HVCs in Complexes C, A, M, WD, and WB. These HVCs were observed previously with UV spectrographs on board $H S T$ and FUSE, including the FUSE survey of high-velocity O vi (Sembach et al. 2003) and the HST/STIS survey of highvelocity Si III (Shull et al. 2009; CSG09). Previously, Mrk 290 was observed with FUSE and HST/GHRS (Wakker et al. 1999; Gibson et al. 2001; CSG03; CSG07). Mrk 817 was observed with HST/GHRS and FUSE (Gibson et al. 2001; CSG03; Fox et al. 2004, 2006). PG 1259+593 was observed with HST/STIS and FUSE (Richter et al. 2001; CSG03; CSG07; Sembach et al. 2004; Fox et al. 2004, 2006). Mrk 876 was observed with HST/ GHRS and FUSE (Gibson et al. 2001; CSG03; CSG07; Fox et al. 2004; Sembach et al. 2003).

Table 1 lists the relevant COS observational parameters of the current COS program. Our targets were observed in both the G130M (1134-1480 $\AA$ ) and G160M (1400-1796 $\AA$ ) medium-resolution gratings $(R \approx 18,000$, $\Delta v \approx 17 \mathrm{~km} \mathrm{~s}^{-1}$ ). The near-UV-imaging target acquisitions were performed with the MIRRORA/PSA mode; the COS primary science aperture (PSA) $(\sim 2$.'5 diameter) yields good centering to maximize throughput and resolving power. Mrk 817 was observed during two 2009 epochs (August 4 and December 28). The first-epoch observations were part of the Early Release Observations (EROs) program (11505, PI: Noll), and the second-epoch observations were part of a COS GTOs program (11524, PI: Green). The first observations were obtained during the Servicing Mission Orbital Verification (SMOV) period before the instrument

\footnotetext{
4 The National Radio Astronomy Observatory is a facility of the National Science Foundation, operated under a cooperative agreement with Associated Universities, Inc.
} 


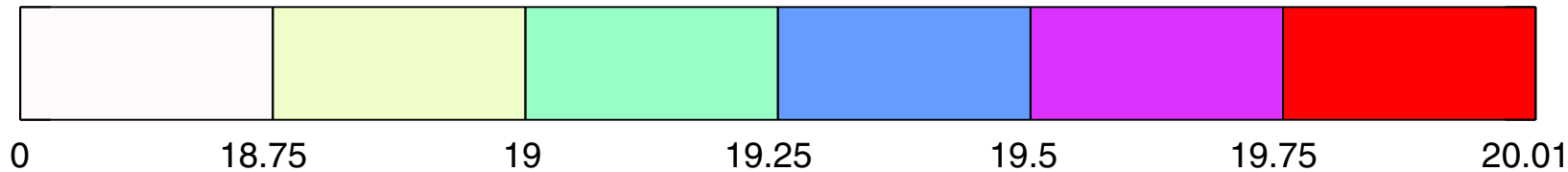

$\log \mathrm{N}(\mathrm{HI})$

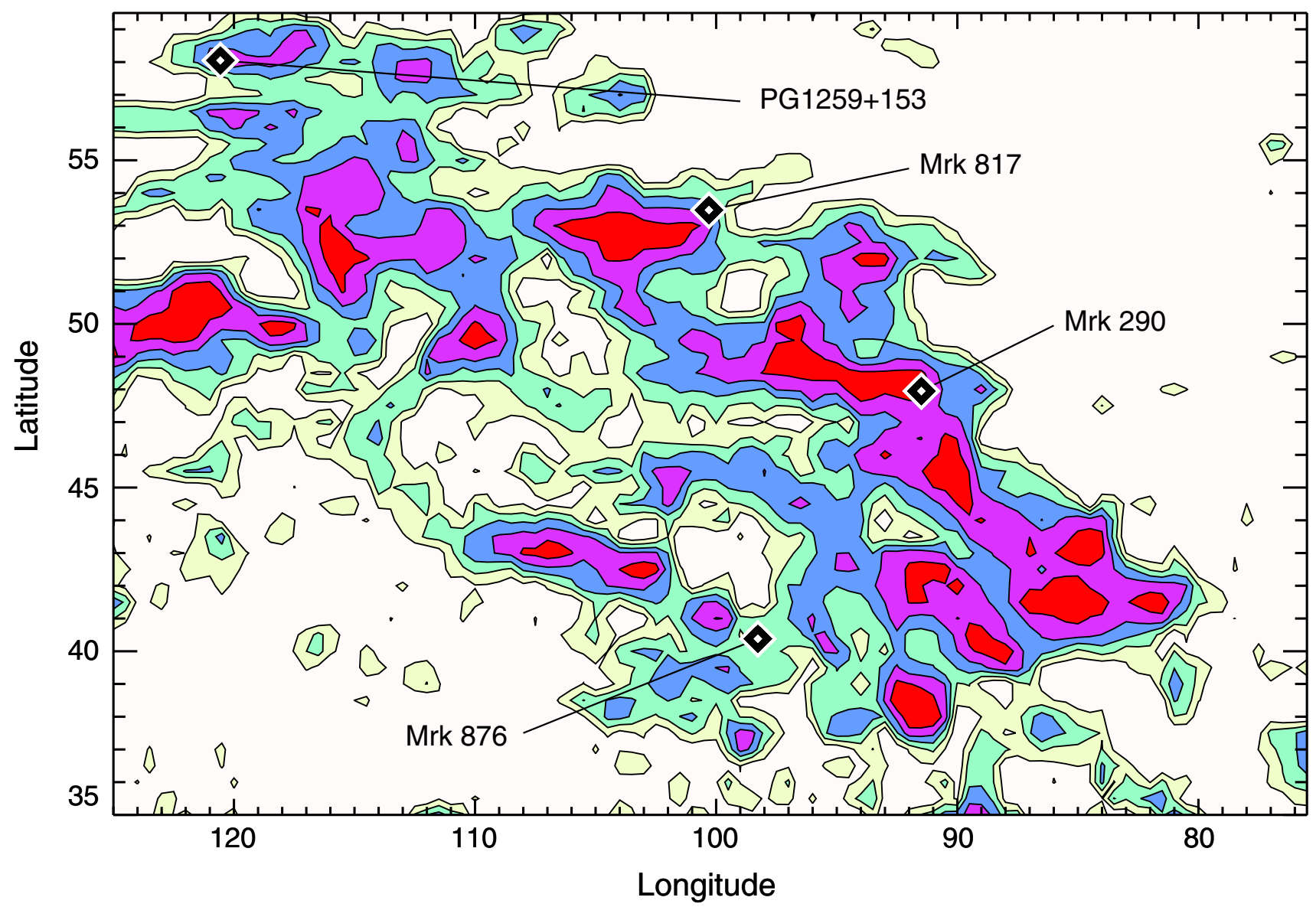

Figure 1. Map of our four AGN targets overlaid on the contour map of $21 \mathrm{~cm}$ emission from the Leiden-Argentine-Bonn (LAB) survey (Kalberla et al. 2005) with $\sim 0.6$ angular resolution on a 0.5 grid in $\ell$ and $b$. Emission is shown over Galactic coordinates between $\ell=75^{\circ}-125^{\circ}$ and $b=35^{\circ}-60^{\circ}$ and over Complex $\mathrm{C}$ velocities between -210 and $-95 \mathrm{~km} \mathrm{~s}^{-1}$. At the $10 \mathrm{kpc}$ distance of Complex C, $1^{\circ}$ corresponds to $175 \mathrm{pc}$.

Table 1

COS Observations

\begin{tabular}{|c|c|c|c|c|c|c|}
\hline Target $^{\mathrm{a}}$ & Coordinates $^{\mathrm{a}}$ & $\begin{array}{l}\text { Program } \\
\text { (s) }\end{array}$ & Grating & $N_{\exp }$ & $\begin{array}{l}t_{\exp } \\
(\mathrm{s})\end{array}$ & $(\mathrm{S} / \mathrm{N})_{\mathrm{res}}^{\mathrm{b}}$ \\
\hline Mrk 817 & $14: 36: 22.1+58: 47: 39$ & 11505,11524 & G130M & 8 & 3426 & 41 \\
\hline$z=0.031455$ & $l=100.30, b=53.48$ & 11505,11524 & G160M & 8 & 3010 & 35 \\
\hline Mrk 290 & $15: 35: 52.3+57: 54: 09$ & 11524 & G130M & 4 & 3857 & 22 \\
\hline$z=0.029577$ & $l=91.49, b=47.95$ & 11524 & G160M & 4 & 4801 & 22 \\
\hline Mrk 876 & $16: 13: 57.2+65: 43: 10$ & 11524,11686 & G130M & 6 & 12580 & 57 \\
\hline$z=0.12900$ & $l=98.27, b=40.38$ & 11686 & G160M & 4 & 11820 & 37 \\
\hline PG $1259+593$ & $13: 01: 13.1+59: 02: 06$ & 11541 & G130M & 4 & 9201 & 35 \\
\hline$z=0.4778$ & $l=120.56, b=58.05$ & 11541 & G160M & 4 & 11169 & 32 \\
\hline
\end{tabular}

Notes.

a AGN target, redshifts, and coordinates: R.A./decl. (2000) and Galactic $\ell$ and $b$.

b Signal-to-noise ratio per resolution element calculated at $1350 \AA$ and $1550 \AA$ for data with G130M (1132-1460 ̊̊) and G160M (1394-1798 $\AA$ ) gratings, respectively. The actual $\mathrm{S} / \mathrm{N}$ values vary throughout each data set, but the quoted values give a sense of relative data quality. See the text for more details.

reached final focus. However, the pre-focus data are largely indistinguishable from that taken after correct focus had been achieved except in the case of the very narrowest of absorp- tion features $(\Delta \lambda \lesssim 0.1 \AA)$, none of which are analyzed in this work. Another symptom unique to the early SMOV data was the "divot and clod" feature which occurred near the blue 


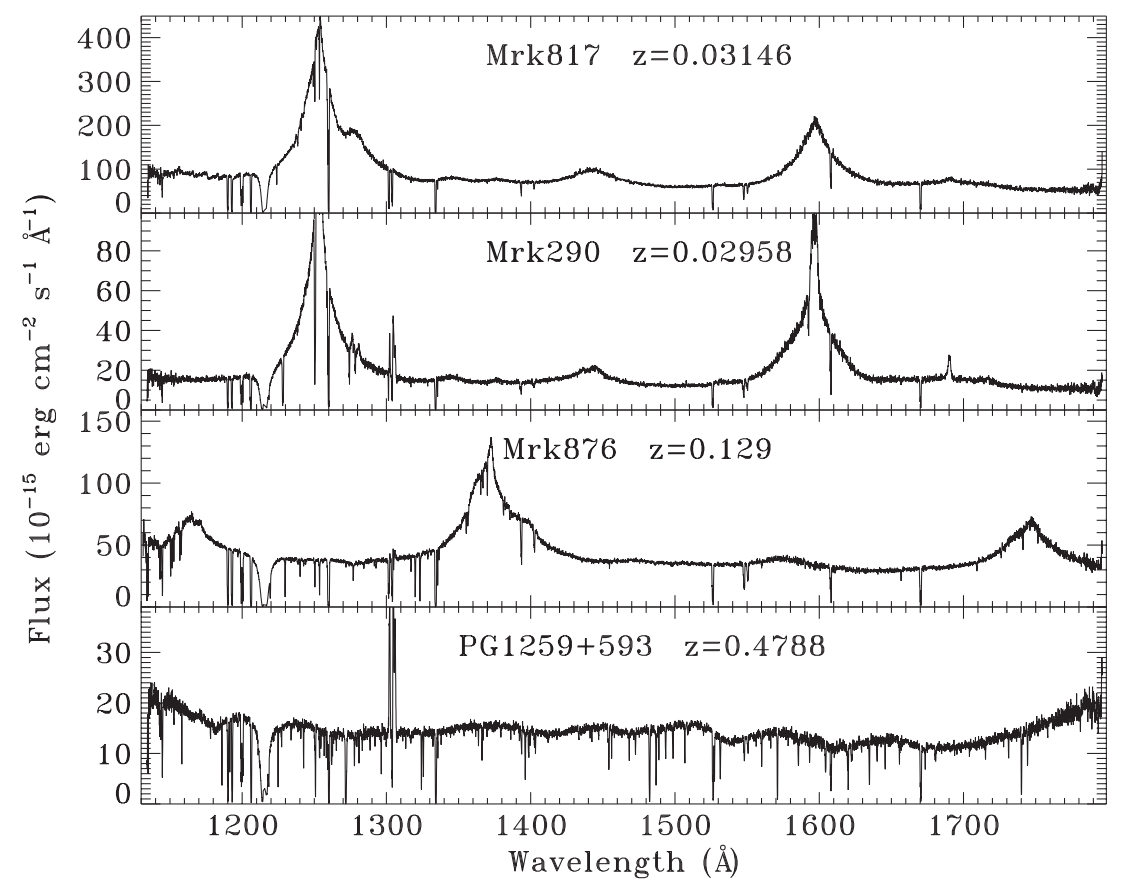

Figure 2. Combined G130M/G160M spectra from HST/COS of the four AGN targets behind Complex C over the wavelength range $1135-1796 \AA$. Note the prominent broad emission lines, Ly $\alpha \lambda 1216$, Si IV/O IV] $\lambda 1400$, C IV $\lambda 1549$, together with numerous narrow absorption lines from the ISM and IGM. Fluxes are in units of $10^{-15} \mathrm{erg} \mathrm{cm}^{-2} \mathrm{~s}^{-1} \AA^{-1}$.

Table 2

Gaussian Fits ${ }^{\mathrm{a}}$ to H I Column Densities

\begin{tabular}{|c|c|c|c|c|}
\hline Target & $\begin{array}{c}\left\langle V_{\mathrm{LSR}}\right\rangle \\
\left(\mathrm{km} \mathrm{s}^{-1}\right)\end{array}$ & $\begin{array}{l}\text { Height } \\
\left(T_{b}(\mathrm{~K})\right)\end{array}$ & $\begin{array}{l}\text { FWHM } \\
\left(\mathrm{km} \mathrm{s}^{-1}\right)\end{array}$ & $\begin{array}{c}N_{\mathrm{H}} \\
\text { (in } 10^{18} \mathrm{~cm}^{-2} \text { ) }\end{array}$ \\
\hline Mrk 817 & $-108.0 \pm 0.12$ & $0.594 \pm 0.0055$ & $27.5 \pm 0.3$ & $31.7 \pm 0.5$ \\
\hline \multirow[t]{3}{*}{ Mrk 290} & $-89.8 \pm 1.2$ & $0.310 \pm 0.030$ & $22.4 \pm 1.3$ & $13.5 \pm 1.5$ \\
\hline & $-113.6 \pm 0.5$ & $0.583 \pm 0.021$ & $25.7 \pm 2.8$ & $29.1 \pm 3.3$ \\
\hline & $-136.3 \pm 0.26$ & $1.608 \pm 0.037$ & $22.2 \pm 0.3$ & $69.4 \pm 1.8$ \\
\hline \multirow[t]{2}{*}{ Mrk 876} & $-131.4 \pm 0.3$ & $0.381 \pm 0.006$ & $33.4 \pm 0.7$ & $24.7 \pm 0.7$ \\
\hline & $-175.5 \pm 0.8$ & $0.116 \pm 0.008$ & $22.7 \pm 1.9$ & $5.1 \pm 0.5$ \\
\hline \multirow[t]{2}{*}{ PG $1259+593$} & $-126.4 \pm 0.1$ & $0.891 \pm 0.061$ & $12.0 \pm 0.5$ & $20.7 \pm 1.6$ \\
\hline & $-129.1 \pm 0.1$ & $1.558 \pm 0.062$ & $24.1 \pm 0.3$ & $73.0 \pm 3.1$ \\
\hline
\end{tabular}

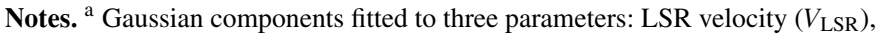
height $\left(T_{b}\right)$, and FWHM, from which we derive column density, $N_{\mathrm{H}_{\mathrm{I}}}=$ $\left(1.823 \times 10^{18} \mathrm{~cm}^{-2}\right)$ (height) $(\mathrm{FWHM})(1.065)$, for height in units of $T_{b}(\mathrm{~K})$ and FWHM in $\mathrm{km} \mathrm{s}^{-1}$.

end of each detector segment; charge resulting from photons hitting one part of the detector appeared in a different part. The high voltage on the detectors was reduced on 2009 August 4, and the instrumental feature disappeared. For the Mrk 817 ERO observations, the divot and clod were carefully fitted with Gaussian profiles and normalized to the flux level in adjacent portions of the spectrum. The observing time was approximately equal at both epochs, but the AGN continuum flux decreased by a factor of 1.4 in the interim. Data from the second epoch were scaled multiplicatively to the flux level of the 2009 August observations. A full discussion of the Mrk 817 data appears in Winter et al. (2011). Targets Mrk 290 and PG 1259+593 were both observed as part of COS/GTO programs 11524 (2009 October 29) and 11541 (2010 April 15), respectively. Mrk 876 was observed (2009 April 8-10) in our COS/GTO program and as a Guest Observer target (11686, PI: Arav). The observations were taken within two days of each other.

After retrieval from the Space Telescope Science Institute $(\mathrm{STScI})$, all data were reduced with the COS calibration

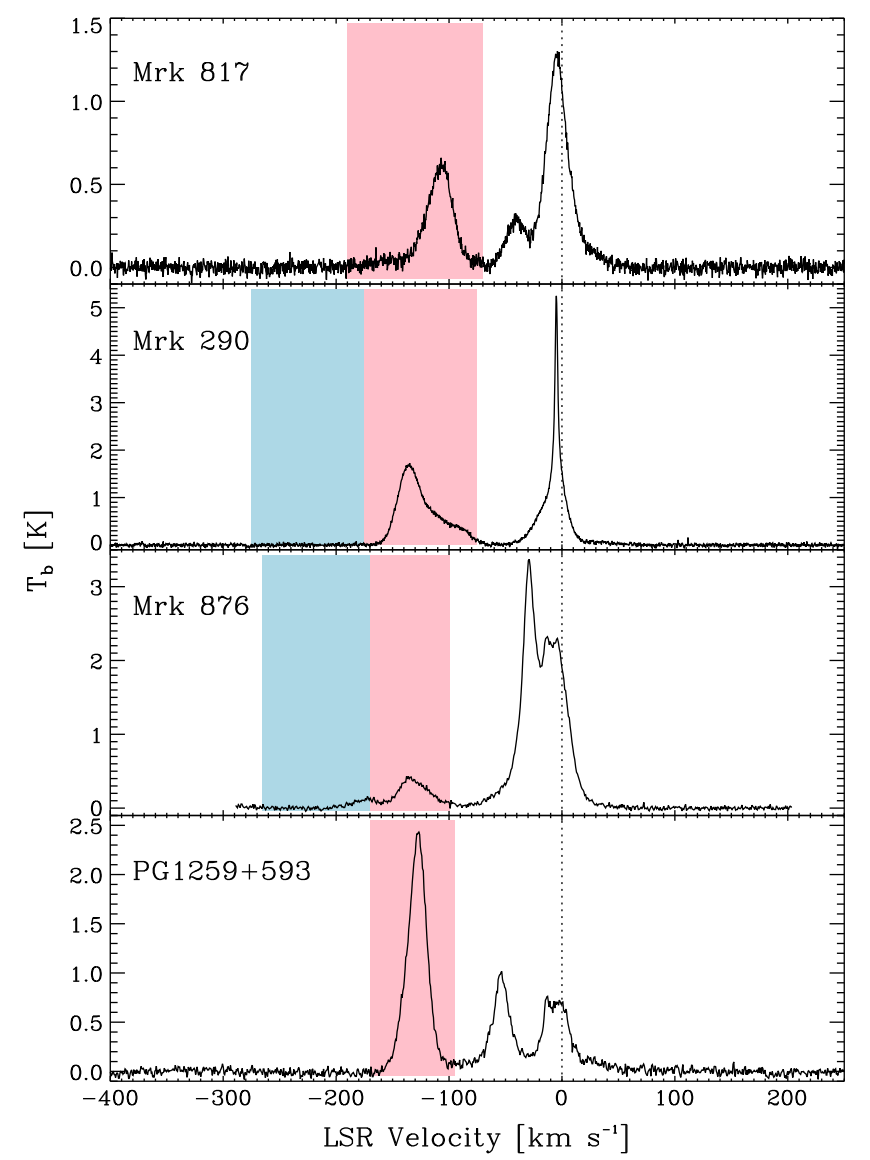

Figure 3. Spectra of $21 \mathrm{~cm}$ emission toward our four target AGNs, taken with the GBT. Velocity ranges in pink wash (Complex $\mathrm{C}$ ) and blue wash (highervelocity gas) show where we detected high-velocity UV absorption with COS: $\operatorname{Mrk} 817$ ( -190 to $\left.-70 \mathrm{~km} \mathrm{~s}^{-1}\right)$, Mrk $290\left(-175\right.$ to $-75 \mathrm{~km} \mathrm{~s}^{-1}$ and -275 to $\left.-175 \mathrm{~km} \mathrm{~s}^{-1}\right)$, Mrk $876\left(-170\right.$ to $-100 \mathrm{~km} \mathrm{~s}^{-1}$ and -265 to $\left.-170 \mathrm{~km} \mathrm{~s}^{-1}\right)$, and PG $1259+593$ ( -170 to $\left.-95 \mathrm{~km} \mathrm{~s}^{-1}\right)$. 
pipeline, $\mathrm{CALCOS}^{5}$ version 2.11f. The analog nature of the COS micro-channel plate makes it susceptible to temperature changes. Electronically injected pulses (stims) at opposite corners of the detector allow for the tracking and correction of any drift (wavelength zero point) and/or stretch (dispersion solution) of the recorded data location as a function of temperature. Flat fielding, alignment, and co-addition of the processed COS exposures were carried out using IDL routines developed by the COS GTO team specifically for COS far-UV data. ${ }^{6}$ The data were corrected for narrow instrumental features arising from shadows from the ion repeller grid wires. We aligned each exposure by cross-correlating strong ISM features and interpolated the aligned exposures onto a common wavelength scale. Wavelength shifts were typically on the order of a resolution element $\left(\sim 0.07 \AA, 17 \mathrm{~km} \mathrm{~s}^{-1}\right)$ or less. The co-added flux at each wavelength was taken to be the exposure-weighted mean of flux in each exposure.

To transfer the COS data to the $V_{\mathrm{LSR}}$ scale of the $21 \mathrm{~cm}$ data, we first aligned the interstellar absorption features in velocity space. Next we chose between 8 and 12 clearly defined HVC absorption features, found the velocity at which their fluxes were minimized, and took the mean. This mean velocity was subtracted from the velocity of the H i $21 \mathrm{~cm} \mathrm{HVC}$ emission peak, and the resulting difference was used to shift the COS data to align with the $21 \mathrm{~cm}$ data. To quantify the quality of the combined data, we identify line-free continuum regions at various wavelengths, smooth the data by the sevenpixel resolution element, and define $(\mathrm{S} / \mathrm{N})_{\text {res }}=$ mean(flux)/ stddev(flux). The $\mathrm{S} / \mathrm{N}$ varies across the wavelength range, but representative values in G130M and G160M data are shown in Table 1.

Previous observations from FUSE and HST/GHRS and HST/ STIS (CSG03; CSG07; Fox et al. 2004, 2006) were reported on all four sight lines (Mrk 290, Mrk 817, Mrk 876, PG 1259). We included FUSE data (HVC lines of $\mathrm{C}$ III and $\mathrm{O}$ VI) and re-measured $\mathrm{O}$ VI $\lambda 1031.93$, using the velocity range defined by the HVC absorbers measured by COS. With the higher $\mathrm{S} / \mathrm{N}$, these spectra provide better definition of the extent of high-velocity UV absorption. Tables 3-6 provide the equivalent widths, $W_{\lambda}$, absorption-line data, and derived column densities. Our error bars include statistical fluctuations in the measurement of equivalent widths and systematic errors arising from variations in the continuum placement and HVC velocity range of integration. Our measurements of equivalent widths vary the continuum placement by one standard deviation in continuum flux and adjust the velocity range of HVC absorption by $10 \mathrm{~km} \mathrm{~s}^{-1}$. These errors are then added in quadrature to produce $1 \sigma$ confidence limits on our apparent optical depth (AOD) measurements.

The HST/COS data on $\mathrm{P}_{\text {II }} \lambda 1152.82$ and the $\mathrm{N}_{\mathrm{I}}$ triplet $\lambda 1199.54,1200.22,1200.70$ (toward Mrk 817, Mrk 876, PG 1259) are much better determined than the FUSE upper limits. Other lines in common include Fe II $\lambda 1143.22$, 1144.94. The complex line profiles of S II and Si II are greatly improved in quality, particularly the S II lines toward Mrk 876 and Mrk 290. We are also able to provide reliable measurements of $\mathrm{N}_{\mathrm{I}}, \mathrm{P}_{\mathrm{II}}$, Al II, and key high ions (C IV, Si IV, N v).

\footnotetext{
5 See the HST Cycle 18 COS Instrument Handbook for more details: http://www.stsci.edu/hst/cos/documents/handbooks/current/cos_cover.html. 6 See http://casa.colorado.edu/ danforth/costools.html for our co-addition and flat-fielding algorithm, which was further described in Danforth et al. (2010).
}

Table 3

HVC Measurements ${ }^{\mathrm{a}}$ (Mrk 817)

\begin{tabular}{|c|c|c|c|c|c|}
\hline Ion & $\begin{array}{l}\lambda_{0} \\
(\AA)\end{array}$ & $\begin{array}{c}V_{c} \\
\left(\mathrm{~km} \mathrm{~s}^{-1}\right)\end{array}$ & $\begin{array}{c}b_{\text {width }} \\
\left(\mathrm{km} \mathrm{s}^{-1}\right)\end{array}$ & $\begin{array}{c}W_{\lambda} \\
(\mathrm{m} \AA)\end{array}$ & $\begin{array}{c}\log N_{a} \\
\left(N \text { in } \mathrm{cm}^{-2}\right)\end{array}$ \\
\hline S II & 1259.51 & $-104 \pm 11$ & $25 \pm 7$ & $42 \pm 7$ & $14.29_{-0.08}^{+0.08}$ \\
\hline $\mathrm{S}_{\text {II }}$ & 1253.80 & $-102 \pm 7$ & $27 \pm 9$ & $19 \pm 5$ & $14.12_{-0.12}^{+0.12}$ \\
\hline S II & 1250.57 & $-123 \pm 1$ & $49 \pm 14$ & $27 \pm 8$ & $14.57_{-0.13}^{+0.15}$ \\
\hline Si II & 1526.70 & $-121 \pm 5$ & $43 \pm 9$ & $364 \pm 66$ & $14.37_{-0.07}^{+0.08}$ \\
\hline Si II & 1304.37 & $-114 \pm 6$ & $38 \pm 6$ & $241 \pm 39$ & $14.47_{-0.05}^{+0.08}$ \\
\hline Si II & 1193.28 & $-116 \pm 6$ & $41 \pm 7$ & $338 \pm 61$ & $14.08_{-0.07}^{+0.09}$ \\
\hline Si II & 1190.41 & $-116 \pm 5$ & $40 \pm 8$ & $295 \pm 57$ & $14.23_{-0.07}^{+0.10}$ \\
\hline Fe II & 1608.45 & $-113 \pm 7$ & $38 \pm 6$ & $173 \pm 32$ & $14.23_{-0.06}^{+0.10}$ \\
\hline Fe II & 1144.93 & $-113 \pm 6$ & $40 \pm 7$ & $117 \pm 25$ & $14.19_{-0.09}^{+0.11}$ \\
\hline Fe II & 1143.22 & $-117 \pm 2$ & $50 \pm 17$ & $49 \pm 14$ & $14.40_{-0.12}^{+0.17}$ \\
\hline $\mathrm{C}$ II ${ }^{*}$ & 1335.66 & $-141 \pm 5$ & $55 \pm 16$ & $31 \pm 15$ & $14.22_{-0.19}^{+0.26}$ \\
\hline $\mathrm{C}_{\mathrm{II}}$ & 1334.53 & $-121 \pm 5$ & $43 \pm 7$ & $431 \pm 75$ & $14.90_{-0.12}^{+0.12}$ \\
\hline $\mathrm{N}_{\mathrm{I}}$ & 1200.71 & $-131 \pm 1$ & $42 \pm 9$ & $284 \pm 44$ & $14.95_{-0.04}^{+0.05}$ \\
\hline $\mathrm{NI}_{\mathrm{I}}$ & 1200.22 & $-138 \pm 2$ & $47 \pm 11$ & $284 \pm 68$ & $14.65_{-0.08}^{+0.12}$ \\
\hline $\mathrm{N}_{\mathrm{I}}$ & 1199.54 & $-109 \pm 5$ & $36 \pm 6$ & $141 \pm 27$ & $14.06_{-0.07}^{+0.10}$ \\
\hline O I & 1302.16 & $-119 \pm 5$ & $42 \pm 7$ & $337 \pm 57$ & $14.98_{-0.06}^{+0.07}$ \\
\hline Al II & 1670.78 & $-119 \pm 6$ & $40 \pm 7$ & $404 \pm 65$ & $13.23_{-0.06}^{+0.07}$ \\
\hline $\mathrm{P}_{\text {II }}$ & 1152.82 & $-117 \pm 8$ & $37 \pm 9$ & $23 \pm 6$ & $12.95_{-0.17}^{+0.09}$ \\
\hline C IV & 1550.78 & $-107 \pm 9$ & $37 \pm 4$ & $100 \pm 24$ & $13.77_{-0.09}^{+0.14}$ \\
\hline C IV & 1548.20 & $-107 \pm 7$ & $38 \pm 6$ & $170 \pm 41$ & $13.75_{-0.09}^{+0.14}$ \\
\hline $\mathrm{N} \mathrm{v}$ & 1242.80 & $-119 \pm 5$ & $52 \pm 10$ & $13 \pm 4$ & $13.08_{-0.15}^{+0.12}$ \\
\hline $\mathrm{N} \mathrm{v}$ & 1238.82 & $-109 \pm 7$ & $34 \pm 10$ & $31 \pm 8$ & $13.16_{-0.13}^{+0.10}$ \\
\hline O VI & 1031.93 & $-115 \pm 7$ & $43 \pm 7$ & $103 \pm 27$ & $14.05_{-0.09}^{+0.16}$ \\
\hline Si III & 1206.50 & $-118 \pm 6$ & $42 \pm 8$ & $355 \pm 67$ & $13.74_{-0.13}^{+0.15}$ \\
\hline Si IV & 1402.77 & $-106 \pm 11$ & $37 \pm 5$ & $56 \pm 16$ & $13.15_{-0.12}^{+0.15}$ \\
\hline Si IV & 1393.76 & $-109 \pm 8$ & $39 \pm 9$ & $91 \pm 25$ & $13.08_{-0.12}^{+0.13}$ \\
\hline
\end{tabular}

Notes. ${ }^{\text {a }}$ Table lists data for measured HVC absorption lines, including ion and rest wavelength, HVC velocity centroid $\left(V_{c}\right)$, line width $\left(b_{\text {width }}\right)$, equivalent width $\left(W_{\lambda}\right)$, and column density $\left(N_{a}\right)$ from AOD integration. Error bars are $1 \sigma$, including both statistical errors in measurement and systematic effects of continuum placement and velocity range. For the $\mathrm{HVC}$ at $\left\langle V_{\mathrm{LSR}}\right\rangle=$ $-108 \mathrm{~km} \mathrm{~s}^{-1}$ our GBT measurements (Table 2) give $\log N_{\mathrm{H}_{\mathrm{I}}}=19.50 \pm 0.01$. Wakker et al. (2003) quote $\log N_{\mathrm{H}_{\mathrm{I}}}=19.51 \pm 0.01$.

\subsection{GBT Observations}

The $21 \mathrm{~cm} \mathrm{Hi}$ data used here were all obtained with the Robert C. Byrd GBT of the NRAO at an angular resolution of $9^{\prime}$. The spectrum for Mrk 876 is that published by Wakker et al. (2011), the spectrum toward PG 1259+593 was obtained from archival GBT data, and new observations were made for Mrk 817 and Mrk 290. In all cases, the data were reduced, calibrated, and corrected for stray radiation following the method outlined in Blagrave et al. (2010) and A. Boothroyd et al. (2011, in preparation). This technique has been shown to produce $21 \mathrm{~cm}$ spectra of HVCs, with errors of a few percent in total $N_{\mathrm{HI}}$ limited by noise and residual instrumental baseline effects. We fitted a second-order polynomial to emission-free regions of each spectrum and smoothed the spectra to produce the following velocity resolution and rms noise: Mrk 876 (0.81 km s$\left.{ }^{-1}, 19 \mathrm{mK}\right)$; PG 1259+593 (0.64 km s$\left.{ }^{-1}, 30 \mathrm{mK}\right)$; Mrk 817 (0.32 km s$\left.{ }^{-1}, 27 \mathrm{mK}\right)$; and Mrk $290\left(0.32 \mathrm{~km} \mathrm{~s}^{-1}\right.$, $25 \mathrm{mK})$.

Table 2 presents Gaussian fits to the $21 \mathrm{~cm}$ HVC components, including their velocities, widths, and $\mathrm{H}$ I column densities. We calculated H I column densities for the HVCs in the optically 
Table 4

HVC Measurements (Mrk 290)

\begin{tabular}{|c|c|c|c|c|c|}
\hline Ion & $\begin{array}{l}\lambda_{0} \\
(\AA)\end{array}$ & $\begin{array}{c}V_{c} \\
\left(\mathrm{~km} \mathrm{~s}^{-1}\right)\end{array}$ & $\begin{array}{c}b_{\text {width }} \\
\left(\mathrm{km} \mathrm{s}^{-1}\right)\end{array}$ & $\begin{array}{c}W_{\lambda} \\
(\mathrm{m} \AA)\end{array}$ & $\begin{array}{c}\log N_{a} \\
\left(N \text { in } \mathrm{cm}^{-2}\right)\end{array}$ \\
\hline \multicolumn{6}{|c|}{$v_{\mathrm{LSR}} \approx-120 \mathrm{~km} \mathrm{~s}^{-1}$ absorber $^{\mathrm{a}}$} \\
\hline S II & 1259.51 & $-123 \pm 5$ & $32 \pm 5$ & $55 \pm 6$ & $14.43_{-0.04}^{+0.05}$ \\
\hline S II & 1253.80 & $-115 \pm 8$ & $35 \pm 10$ & $11 \pm 2$ & $13.93_{-0.07}^{+0.06}$ \\
\hline S II & 1250.57 & $-104 \pm 12$ & $34 \pm 4$ & $83 \pm 35$ & $15.14_{-0.19}^{+0.26}$ \\
\hline Si II & 1526.70 & $-123 \pm 2$ & $37 \pm 9$ & $317 \pm 64$ & $14.32_{-0.06}^{+0.08}$ \\
\hline Si II & 1304.37 & $-118 \pm 4$ & $34 \pm 6$ & $207 \pm 33$ & $14.40_{-0.04}^{+0.09}$ \\
\hline Si II & 1193.28 & $-114 \pm 5$ & $34 \pm 8$ & $269 \pm 59$ & $14.16_{-0.07}^{+0.13}$ \\
\hline Fe II & 1608.45 & $-121 \pm 3$ & $35 \pm 7$ & $180 \pm 28$ & $14.26_{-0.05}^{+0.08}$ \\
\hline Fe II & 1144.93 & $-128 \pm 4$ & $38 \pm 9$ & $141 \pm 30$ & $14.30_{-0.09}^{+0.11}$ \\
\hline $\mathrm{C}$ II* & 1335.66 & $-120 \pm 39$ & $43 \pm 17$ & $<15$ & $<14.35(3 \sigma)$ \\
\hline $\mathrm{C}_{\mathrm{II}}$ & 1334.53 & $-122 \pm 3$ & $40 \pm 12$ & $389 \pm 11$ & $14.85_{-0.49}^{+0.50}$ \\
\hline $\mathrm{N}_{\mathrm{I}}$ & 1200.71 & $-127 \pm 1$ & $34 \pm 7$ & $212 \pm 30$ & $14.82_{-0.04}^{+0.06}$ \\
\hline $\mathrm{N}_{\mathrm{I}}$ & 1200.22 & $-135 \pm 4$ & $39 \pm 11$ & $210 \pm 66$ & $14.51_{-0.14}^{+0.19}$ \\
\hline N I & 1199.54 & $-119 \pm 3$ & $36 \pm 8$ & $144 \pm 26$ & $14.06_{-0.05}^{+0.10}$ \\
\hline $\mathrm{OI}_{\mathrm{I}}$ & 1302.16 & $-130 \pm 5$ & $26 \pm 4$ & $187 \pm 4$ & $14.64_{-0.02}^{+0.03}$ \\
\hline Al II & 1670.78 & $-124 \pm 4$ & $38 \pm 10$ & $339 \pm 83$ & $13.11_{-0.09}^{+0.12}$ \\
\hline P II & 1152.82 & $-120 \pm 25$ & $45 \pm 10$ & $16 \pm 4$ & $12.83_{-0.18}^{+0.22}$ \\
\hline C IV & 1550.78 & $-118 \pm 3$ & $38 \pm 12$ & $104 \pm 31$ & $13.79_{-0.13}^{+0.13}$ \\
\hline C IV & 1548.20 & $-115 \pm 5$ & $37 \pm 11$ & $177 \pm 54$ & $13.77_{-0.13}^{+0.15}$ \\
\hline $\mathrm{Nv}$ & 1242.80 & $-131 \pm 17$ & $50 \pm 20$ & $8 \pm 4$ & $12.89_{-0.18}^{+0.55}$ \\
\hline $\mathrm{N} \mathrm{v}$ & 1238.82 & $-105 \pm 1$ & $37 \pm 7$ & $18 \pm 7$ & $12.98_{-0.15}^{+0.22}$ \\
\hline O VI & 1031.93 & $-124 \pm 1$ & $38 \pm 11$ & $113 \pm 38$ & $14.10_{-0.14}^{+0.15}$ \\
\hline Si III & 1206.50 & $-120 \pm 1$ & $40 \pm 12$ & $345 \pm 96$ & $13.73_{-0.15}^{+0.18}$ \\
\hline Si IV & 1402.77 & $-116 \pm 3$ & $39 \pm 11$ & $70 \pm 19$ & $13.26_{-0.10}^{+0.16}$ \\
\hline Si IV & 1393.76 & $-120 \pm 2$ & $41 \pm 11$ & $108 \pm 33$ & $13.17_{-0.12}^{+0.15}$ \\
\hline \multicolumn{6}{|c|}{$v_{\mathrm{LSR}} \approx-220 \mathrm{~km} \mathrm{~s}^{-1}$ absorber $^{\mathrm{b}}$} \\
\hline P II & 1152.82 & $-225 \pm 12$ & $56 \pm 5$ & $<15$ & $<13.19(3 \sigma)$ \\
\hline C IV & 1550.78 & $-224 \pm 1$ & $33 \pm 11$ & $71 \pm 17$ & $13.60_{-0.12}^{+0.10}$ \\
\hline C IV & 1548.20 & $-223 \pm 4$ & $35 \pm 5$ & $110 \pm 14$ & $13.51_{-0.04}^{+0.08}$ \\
\hline O VI & 1031.93 & $-219 \pm 8$ & $33 \pm 4$ & $53 \pm 14$ & $13.75_{-0.22}^{+0.15}$ \\
\hline Si IV & 1402.77 & $-218 \pm 2$ & $33 \pm 12$ & $35 \pm 8$ & $12.95_{-0.13}^{+0.12}$ \\
\hline Si IV & 1393.76 & $-218 \pm 7$ & $32 \pm 7$ & $69 \pm 12$ & $12.96_{-0.09}^{+0.07}$ \\
\hline Si III & 1206.50 & $-211 \pm 7$ & $31 \pm 5$ & $180 \pm 38$ & $13.15_{-0.08}^{+0.10}$ \\
\hline
\end{tabular}

Notes.

a Table lists data for measured HVC absorption lines, including ion and rest wavelength, HVC velocity centroid $\left(V_{c}\right)$, line width $\left(b_{\text {width }}\right)$, equivalent width $\left(W_{\lambda}\right)$, and column density $\left(N_{a}\right)$ from AOD integration. Error bars are $1 \sigma$, including both statistical errors in measurement and systematic effects of continuum placement and velocity range. For the composite UV absorber at $\left\langle V_{\mathrm{LSR}}\right\rangle=-120 \mathrm{~km} \mathrm{~s}^{-1}$, our GBT measurements (Table 2) give $\log N_{\mathrm{H}_{\mathrm{I}}}=$ $20.05 \pm 0.02$ for components at $V_{\mathrm{LSR}}=-136,-113$, and $-90 \mathrm{~km} \mathrm{~s}^{-1}$. Wakker et al. (2003) measure a combined $\log N_{\mathrm{H}_{\mathrm{I}}}=20.10 \pm 0.02$ for HVCs at $-134 \mathrm{~km} \mathrm{~s}^{-1}$ and $-105 \mathrm{~km} \mathrm{~s}^{-1}$.

${ }^{\mathrm{b}}$ For the UV-only absorber at $\left\langle V_{\mathrm{LSR}}\right\rangle-220 \mathrm{~km} \mathrm{~s}^{-1}$, Wakker et al. (2003) find no H I emission to a limit $\log N_{\mathrm{H}_{\mathrm{I}}}<18.3$. Our GBT limit over the range $(-275$ to $-175 \mathrm{~km} \mathrm{~s}^{-1}$ ) is $\log N_{\mathrm{H}_{\mathrm{I}}}<18.0$ (at $4 \sigma$ ).

thin assumption (see the footnote to Table 2), which introduces a negligible error for the weak HVC lines. The free parameters are the line centers $\left(V_{\mathrm{LSR}}\right)$ of the Gaussians, and their height (brightness temperature $T_{b}$ ) and FWHM in $\mathrm{km} \mathrm{s}^{-1}$. We detected $\mathrm{Hi}$ emission from all HVCs seen in the COS data, except for the most negative velocity component $\left(V_{\mathrm{LSR}}=-275 \mathrm{~km} \mathrm{~s}^{-1}\right.$ to $-175 \mathrm{~km} \mathrm{~s}^{-1}$ ) toward Mrk 290. For this range, we estimate
Table 5

HVC Measurements ${ }^{\mathrm{a}}$ (Mrk 876)

\begin{tabular}{|c|c|c|c|c|c|}
\hline Ion & $\begin{array}{l}\lambda_{0} \\
(\AA)\end{array}$ & $\begin{array}{c}V_{c} \\
\left(\mathrm{~km} \mathrm{~s}^{-1}\right)\end{array}$ & $\begin{array}{c}b_{\text {width }} \\
\left(\mathrm{km} \mathrm{s}^{-1}\right)\end{array}$ & $\begin{array}{c}W_{\lambda} \\
(\mathrm{m} \AA)\end{array}$ & $\begin{array}{c}\log N_{a} \\
\left(N \text { in } \mathrm{cm}^{-2}\right)\end{array}$ \\
\hline \multicolumn{6}{|c|}{$v_{\mathrm{LSR}} \approx-133 \mathrm{~km} \mathrm{~s}^{-1}$ absorber $^{\mathrm{a}}$} \\
\hline S II & 1259.51 & $-132 \pm 3$ & $25 \pm 10$ & $37 \pm 13$ & $14.24_{-0.13}^{+0.16}$ \\
\hline $\mathrm{S}_{\text {II }}$ & 1253.80 & $-133 \pm 2$ & $24 \pm 11$ & $25 \pm 8$ & $14.25_{-0.14}^{+0.13}$ \\
\hline $\mathrm{S}_{\text {II }}$ & 1250.57 & $-137 \pm 3$ & $24 \pm 10$ & $16 \pm 5$ & $14.37_{-0.13}^{+0.14}$ \\
\hline Si II & 1526.70 & $-135 \pm 1$ & $26 \pm 10$ & $259 \pm 86$ & $14.30_{-0.08}^{+0.14}$ \\
\hline Si II & 1304.37 & $-134 \pm 1$ & $26 \pm 11$ & $191 \pm 65$ & $14.41_{-0.09}^{+0.16}$ \\
\hline Si II & 1193.28 & $-134 \pm 1$ & $28 \pm 12$ & $242 \pm 100$ & $13.97_{-0.12}^{+0.19}$ \\
\hline Si II & 1190.41 & $-133 \pm 1$ & $27 \pm 12$ & $227 \pm 90$ & $14.19_{-0.11}^{+0.18}$ \\
\hline Fe II & 1608.45 & $-136 \pm 1$ & $26 \pm 11$ & $168 \pm 56$ & $14.26_{-0.10}^{+0.17}$ \\
\hline Fe II & 1144.93 & $-134 \pm 2$ & $27 \pm 11$ & $118 \pm 43$ & $14.24_{-0.12}^{+0.18}$ \\
\hline Fe II & 1143.22 & $-140 \pm 2$ & $24 \pm 6$ & $34 \pm 6$ & $14.25_{-0.06}^{+0.13}$ \\
\hline Fe II & 1133.67 & $-132 \pm 4$ & $29 \pm 8$ & $31 \pm 13$ & $14.83_{-0.15}^{+0.27}$ \\
\hline $\mathrm{P}_{\mathrm{II}}$ & 1152.82 & $-130 \pm 1$ & $29 \pm 12$ & $24 \pm 10$ & $12.97_{-0.12}^{+0.32}$ \\
\hline $\mathrm{C}_{\mathrm{II}}{ }^{*}$ & 1335.66 & $-137 \pm 7$ & $26 \pm 13$ & $14 \pm 8$ & $13.86_{-0.24}^{+0.21}$ \\
\hline C II & 1334.53 & $-134 \pm 1$ & $28 \pm 13$ & $292 \pm 134$ & $14.79_{-0.16}^{+0.24}$ \\
\hline $\mathrm{N}_{\mathrm{I}}$ & 1200.71 & $-139 \pm 3$ & $26 \pm 10$ & $213 \pm 78$ & $14.99_{-0.11}^{+0.19}$ \\
\hline $\mathrm{N}_{\mathrm{I}}$ & 1200.22 & $-142 \pm 4$ & $26 \pm 11$ & $148 \pm 68$ & $14.36_{-0.23}^{+0.26}$ \\
\hline $\mathrm{N}_{\mathrm{I}}$ & 1199.54 & $-134 \pm 2$ & $24 \pm 9$ & $112 \pm 32$ & $13.97_{-0.08}^{+0.13}$ \\
\hline O I & 1302.16 & $-136 \pm 1$ & $26 \pm 11$ & $206 \pm 79$ & $14.74_{-0.12}^{+0.16}$ \\
\hline $\mathrm{Al}$ II & 1670.78 & $-137 \pm 1$ & $27 \pm 12$ & $280 \pm 105$ & $13.12_{-0.11}^{+0.17}$ \\
\hline C IV & 1550.78 & $-130 \pm 3$ & $29 \pm 13$ & $73 \pm 37$ & $13.63_{-0.18}^{+0.31}$ \\
\hline C IV & 1548.20 & $-129 \pm 2$ & $29 \pm 12$ & $117 \pm 57$ & $13.58_{-0.18}^{+0.29}$ \\
\hline $\mathrm{N} \mathrm{v}$ & 1242.80 & $-135 \pm 1$ & $28 \pm 15$ & $7 \pm 4$ & $12.82_{-0.29}^{+0.18}$ \\
\hline $\mathrm{Nv}$ & 1238.82 & $-139 \pm 2$ & $27 \pm 14$ & $14 \pm 6$ & $12.85_{-0.14}^{+0.23}$ \\
\hline O VI & 1031.93 & $-136 \pm 1$ & $30 \pm 13$ & $86 \pm 43$ & $13.99_{-0.14}^{+0.36}$ \\
\hline Si IV & 1402.77 & $-128 \pm 4$ & $28 \pm 11$ & $48 \pm 20$ & $13.09_{-0.14}^{+0.24}$ \\
\hline Si IV & 1393.76 & $-129 \pm 3$ & $24 \pm 11$ & $91 \pm 39$ & $13.10_{-0.15}^{+0.25}$ \\
\hline Si III & 1206.50 & $-134 \pm 1$ & $29 \pm 12$ & $269 \pm 117$ & $13.72_{-0.14}^{+0.24}$ \\
\hline \multicolumn{6}{|c|}{$v_{\mathrm{LSR}} \approx-190 \mathrm{~km} \mathrm{~s}^{-1}$ absorber $^{\mathrm{b}}$} \\
\hline Si III & 1206.50 & $-198 \pm 8$ & $27 \pm 7$ & $162 \pm 59$ & $13.17_{-0.19}^{+0.21}$ \\
\hline Si II & 1526.70 & $-197 \pm 8$ & $24 \pm 6$ & $129 \pm 42$ & $13.81_{-0.18}^{+0.13}$ \\
\hline Si II & 1304.37 & $-200 \pm 8$ & $31 \pm 6$ & $87 \pm 30$ & $13.92_{-0.15}^{+0.17}$ \\
\hline Si II & 1193.28 & $-196 \pm 8$ & $25 \pm 5$ & $145 \pm 49$ & $13.56_{-0.13}^{+0.21}$ \\
\hline Si II & 1190.41 & $-196 \pm 7$ & $27 \pm 7$ & $118 \pm 42$ & $13.69_{-0.14}^{+0.21}$ \\
\hline Fe II & 1608.45 & $-200 \pm 9$ & $30 \pm 9$ & $77 \pm 32$ & $13.84_{-0.20}^{+0.17}$ \\
\hline Fe II & 1144.93 & $-200 \pm 4$ & $33 \pm 14$ & $40 \pm 21$ & $13.68_{-0.23}^{+0.22}$ \\
\hline O I & 1302.16 & $-199 \pm 7$ & $28 \pm 6$ & $165 \pm 47$ & $14.57_{-0.12}^{+0.15}$ \\
\hline Al II & 1670.78 & $-202 \pm 9$ & $30 \pm 6$ & $151 \pm 54$ & $12.64_{-0.22}^{+0.14}$ \\
\hline C IV & 1550.78 & $-191 \pm 14$ & $18 \pm 3$ & $25 \pm 12$ & $13.13_{-0.12}^{+0.15}$ \\
\hline C IV & 1548.20 & $-192 \pm 10$ & $27 \pm 5$ & $43 \pm 19$ & $13.09_{-0.17}^{+0.25}$ \\
\hline Si IV & 1402.77 & $-188 \pm 20$ & $13 \pm 8$ & $11 \pm 3$ & $12.40_{-0.12}^{+0.25}$ \\
\hline Si IV & 1393.76 & $-195 \pm 6$ & $34 \pm 13$ & $22 \pm 13$ & $12.44_{-0.24}^{+0.31}$ \\
\hline
\end{tabular}

Notes.

a Table lists data for measured HVC absorption lines, including ion and rest wavelength, HVC velocity centroid $\left(V_{c}\right)$, line width $\left(b_{\text {width }}\right)$, equivalent width $\left(W_{\lambda}\right)$, and column density $\left(N_{a}\right)$ from AOD integration. Error bars are $1 \sigma$, including both statistical errors in measurement and systematic effects of continuum placement and velocity range. Our GBT measurements give $\log N_{\mathrm{H}_{\mathrm{I}}}=19.39 \pm 0.01$ and $\log N_{\mathrm{HI}}=18.71 \pm 0.04$ for HVC components at $V_{\mathrm{LSR}}=-131 \mathrm{~km} \mathrm{~s}^{-1}$ and $-175 \mathrm{~km} \mathrm{~s}^{-1}$, respectively (Table 2). Wakker et al. (2003) measure $\log N_{\mathrm{HI}}=19.30 \pm 0.02$ at $V_{\mathrm{LSR}}=-133 \mathrm{~km} \mathrm{~s}^{-1}$.

b For this UV-only HVC, our GBT measurements (Table 2) give $\log N_{\mathrm{HI}_{\mathrm{I}}}=$ $18.71 \pm 0.04$ at $V_{\mathrm{LSR}}=-175 \mathrm{~km} \mathrm{~s}^{-1}$. Wakker et al. (2003) report no $\mathrm{HI}_{\mathrm{I}}$ emission at $-190 \mathrm{~km} \mathrm{~s}^{-1}$, but they find $\log N_{\mathrm{H}_{\mathrm{I}}}=18.67 \pm 0.08$ for a weak component at $V_{\mathrm{LSR}}=-173 \mathrm{~km} \mathrm{~s}^{-1}$. 
Table 6

HVC Measurements $^{\mathrm{a}}(\mathrm{PG} 1259+593)$

\begin{tabular}{|c|c|c|c|c|c|}
\hline Ion & $\begin{array}{l}\lambda_{0} \\
(\AA)\end{array}$ & $\begin{array}{c}V_{c} \\
\left(\mathrm{~km} \mathrm{~s}^{-1}\right)\end{array}$ & $\begin{array}{c}b_{\text {width }} \\
\left(\mathrm{km} \mathrm{s}^{-1}\right)\end{array}$ & $\begin{array}{c}W_{\lambda} \\
(\mathrm{m} \AA)\end{array}$ & $\begin{array}{c}\log N_{a} \\
\left(N \text { in } \mathrm{cm}^{-2}\right)\end{array}$ \\
\hline $\mathrm{S}_{\text {II }}$ & 1259.51 & $-127 \pm 6$ & $23 \pm 7$ & $38 \pm 10$ & $14.27_{-0.11}^{+0.11}$ \\
\hline $\mathrm{S}_{\text {II }}$ & 1253.80 & $-128 \pm 5$ & $25 \pm 9$ & $30 \pm 8$ & $14.35_{-0.10}^{+0.15}$ \\
\hline $\mathrm{S}_{\text {II }}$ & 1250.57 & $-120 \pm 8$ & $18 \pm 1$ & $22 \pm 5$ & $14.51_{-0.10}^{+0.11}$ \\
\hline Si II & 1526.70 & $-124 \pm 6$ & $25 \pm 9$ & $188 \pm 62$ & $14.06_{-0.15}^{+0.14}$ \\
\hline Si II & 1304.37 & $-124 \pm 6$ & $22 \pm 8$ & $126 \pm 39$ & $14.17_{-0.14}^{+0.10}$ \\
\hline Si II & 1193.28 & $-121 \pm 7$ & $24 \pm 7$ & $166 \pm 55$ & $13.66_{-0.15}^{+0.19}$ \\
\hline Si II & 1190.41 & $-122 \pm 5$ & $25 \pm 9$ & $160 \pm 56$ & $13.90_{-0.14}^{+0.17}$ \\
\hline Fe II & 1608.45 & $-126 \pm 7$ & $24 \pm 7$ & $123 \pm 34$ & $14.11_{-0.11}^{+0.12}$ \\
\hline Fe II & 1144.93 & $-126 \pm 6$ & $29 \pm 12$ & $97 \pm 39$ & $14.13_{-0.16}^{+0.22}$ \\
\hline Fe II & 1143.22 & $-138 \pm 0$ & $19 \pm 14$ & $26 \pm 5$ & $14.16_{-0.12}^{+0.11}$ \\
\hline P II & 1152.82 & $-130 \pm 3$ & $13 \pm 28$ & $<10$ & $<13.02(3 \sigma)$ \\
\hline $\mathrm{C}_{\mathrm{II}}{ }^{*}$ & 1335.66 & $-140 \pm 13$ & $33 \pm 8$ & $35 \pm 24$ & $14.29_{-0.31}^{+0.34}$ \\
\hline $\mathrm{C}_{\text {II }}$ & 1334.53 & $-122 \pm 5$ & $26 \pm 9$ & $219 \pm 75$ & $14.45_{-0.17}^{+0.23}$ \\
\hline $\mathrm{N}_{\mathrm{I}}$ & 1200.71 & $-130 \pm 1$ & $30 \pm 12$ & $193 \pm 77$ & $14.79_{-0.14}^{+0.21}$ \\
\hline $\mathrm{N}_{\mathrm{I}}$ & 1200.22 & $-140 \pm 3$ & $27 \pm 12$ & $167 \pm 67$ & $14.44_{-0.14}^{+0.23}$ \\
\hline $\mathrm{N}_{\mathrm{I}}$ & 1199.54 & $-127 \pm 5$ & $24 \pm 9$ & $89 \pm 26$ & $13.85_{-0.12}^{+0.12}$ \\
\hline $\mathrm{Al}$ II & 1670.78 & $-127 \pm 5$ & $26 \pm 9$ & $214 \pm 69$ & $12.89_{-0.16}^{+0.13}$ \\
\hline C IV & 1550.78 & $-124 \pm 9$ & $27 \pm 8$ & $25 \pm 10$ & $13.13_{-0.16}^{+0.24}$ \\
\hline C IV & 1548.20 & $-115 \pm 8$ & $23 \pm 7$ & $41 \pm 19$ & $13.08_{-0.15}^{+0.33}$ \\
\hline $\mathrm{Nv}$ & 1242.80 & $-125 \pm 6$ & $31 \pm 13$ & $<14$ & $<13.59(3 \sigma)$ \\
\hline $\mathrm{N} \mathrm{v}$ & 1238.82 & $-122 \pm 9$ & $23 \pm 10$ & $<7$ & $<12.99(3 \sigma)$ \\
\hline O VI & 1031.93 & $-130 \pm 3$ & $27 \pm 8$ & $39 \pm 13$ & $13.58_{-0.12}^{+0.10}$ \\
\hline Si IV & 1402.77 & $-119 \pm 4$ & $25 \pm 11$ & $23 \pm 9$ & $12.75_{-0.16}^{+0.22}$ \\
\hline Si IV & 1393.76 & $-124 \pm 6$ & $26 \pm 8$ & $34 \pm 12$ & $12.64_{-0.13}^{+0.17}$ \\
\hline Si III & 1206.50 & $-124 \pm 6$ & $27 \pm 8$ & $187 \pm 67$ & $13.30_{-0.16}^{+0.24}$ \\
\hline
\end{tabular}

Notes. ${ }^{a}$ Table lists data for measured HVC absorption lines, including ion and rest wavelength, HVC velocity centroid $\left(V_{c}\right)$, line width $\left(b_{\text {width }}\right)$, equivalent width $\left(W_{\lambda}\right)$, and column density $\left(N_{a}\right)$ from AOD integration. Error bars are $1 \sigma$, including both statistical errors in measurement and systematic effects of continuum placement and velocity range. The GBT measurements give $\log N_{\mathrm{HI}}=19.97 \pm 0.02$ for the two-component HVC $\left(-126.4 \mathrm{~km} \mathrm{~s}^{-1}\right.$ and $=129.1 \mathrm{~km} \mathrm{~s}^{-1}$ ) at $\left\langle V_{\mathrm{LSR}}\right\rangle=-128 \mathrm{~km} \mathrm{~s}^{-1}$. Wakker et al. (2003) measure $\log N_{\mathrm{HI}}=19.95 \pm 0.01$ for an HVC at $V_{\mathrm{LSR}}=-128 \mathrm{~km} \mathrm{~s}^{-1}$, and Sembach et al. (2004) adopt $\log N_{\mathrm{HI}}=19.94 \pm 0.06$ for this HVC.

an upper limit from the noise in the $21 \mathrm{~cm}$ spectrum integrated over the velocity range of the HVC.

All these directions were observed previously in the $21 \mathrm{~cm}$ line by Wakker et al. (2001) using the Effelsberg radio telescope (9.7 beam). The GBT data (9'0 beam) have lower noise by factors of two to four, slightly better angular resolution, and better spectral baselines. The higher GBT S/N allows us to fit two components to the HVC toward PG $1259+593$, whereas Wakker et al. (2001) fitted only one. In general, the two telescopes give similar values of $N_{\mathrm{HI}}$, to within the errors, except for the $-136 \mathrm{~km} \mathrm{~s}^{-1}$ component of Mrk 290, where the GBT value is $20 \%$ below that from Effelsberg, and the $-131 \mathrm{~km} \mathrm{~s}^{-1}$ component of Mrk 876, where the GBT value lies $32 \%$ above. In the latter case, the Effelsberg spectrum appears to have been affected by interference. As noted in the captions to Tables 3-6, the total HVC column densities, $\log N_{\mathrm{HI}}$, used in this paper (GBT) are similar to those from Effelsberg (Eff): Mrk 817 (19.50 \pm 0.01 from GBT, $19.51 \pm 0.01$ from Eff); Mrk $290(20.05 \pm 0.01$ from GBT, $20.10 \pm 0.02$ from Eff); Mrk 876 (19.39 \pm 0.02 from GBT, $19.30 \pm 0.02$ from Eff); and
PG $1259+593$ (19.97 \pm 0.02 from GBT, 19.95 \pm 0.01 from Eff). The primary differences are in the emission-line profiles.

\section{SCIENTIFIC RESULTS AND ANALYSIS}

\subsection{Spectra of HVC Absorption Lines}

To illustrate the complexities of identifying an HVC in actual UV absorption-line spectra, it is helpful to show true fluxes versus wavelength before deriving equivalent widths from flux-normalized spectra. The UV spectra of AGNs are rich in interstellar lines of metal ions, but they often contain low-redshift IGM absorbers. The interstellar absorbers include low-velocity gas near the LSR, as well as high-velocity and intermediate-velocity gas.

Figures 4-7 present 12 panel plots showing a standard set of HVC absorbers, ranging from low- to high-ionization states. The velocities of Complex $\mathrm{C}$ absorption are shown in pink wash. Along two sight lines (Mrk 290 and Mrk 876), additional HVCs at higher negative velocity are shown in blue wash. We show selected interstellar spectral features for the four AGN sight lines: absorption lines of eight low-ionization species (C II, N I, O I, S II, Si II, Fe II, Al II, P II) and four more highly ionized species ( $\mathrm{Si}$ III, Si IV, C IV, Nv). We also reanalyzed the O VI data from FUSE, using the velocity ranges of HVC ultraviolet absorption, defined by absorption lines of N I, O I, Si II, S II, Fe II, and Al II. In many panels, we show multiple transitions of the same species, including the N I triplet (1199.550, 1200.223, $1200.710 \AA)$, the S II triplet (1250.584, 1253.811, $1259.510 \AA)$, the doublets of C IV (1548.195, 1550.770 ̊), Si IV (1393.755, $1402.770 \AA)$, and N v (1238.821, $1242.804 \AA)$, two Si II lines $(1190.416,1193.290 \AA)$, and two lines from the $\mathrm{C}$ II ground state (1334.532 $\AA$ ) and $\mathrm{C}_{\text {II }}{ }^{*}$ fine-structure state $(1335.663 \AA)$. One

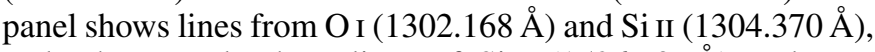
and other panels show lines of Si II (1526.707 $\AA$ ) and Fe II (1143.226, $1144.938 \AA$, and 1608.451 $)$.

The HVC absorption lines are analyzed to provide absorption equivalent width, $W_{\lambda}$, effective line width $\left(b_{\text {width }}\right)$, and column density, $N$, with oscillator strengths from Morton (2003). The column densities were derived using the "AOD" method (Sembach \& Savage 1992), which yields the column density, $N_{a}(v)$, in a given velocity interval. We then derive the total column density by integrating $N_{a}(v)$ over the velocity range of the HVC. A few of the lines (Si III, Al II) are mildly saturated, but we have checked the column densities from the AOD method with the CoGs (curves of growth) for these sight lines, determined from the low ions (CSG03) for accuracy. We believe they are reasonable estimates; further discussion is given in Section 3.4.

The COS line-spread function (LSF) has broad wings with significant power, as documented on the STScI/COS Web site (Kriss 2011). Our COS data-reduction software accounts for the LSF when fitting Voigt profiles to absorption lines, but the LSF is not included in the AOD method for deriving column densities, $N_{a}(v)$, in fixed velocity bins. Thus, some of the increased velocity range could be instrumental in nature, rather than due to the physical nature of the gas itself. However, the differences in velocity and O vi column densities are not large, as we discuss in the notes on individual sight lines (Section 3.5).

Figures 8-11 show "stack plots" of the HVC absorbers, aligned in velocity space using normalized continua. Our previous experience with the COS wavelength scale (Osterman et al. 2011) suggests that differential shifts up to $10 \mathrm{~km} \mathrm{~s}^{-1}$ are possible, relative to the $21 \mathrm{~cm}$ emission data. In general, the HVC absorption in the UV metal lines agrees well with the 

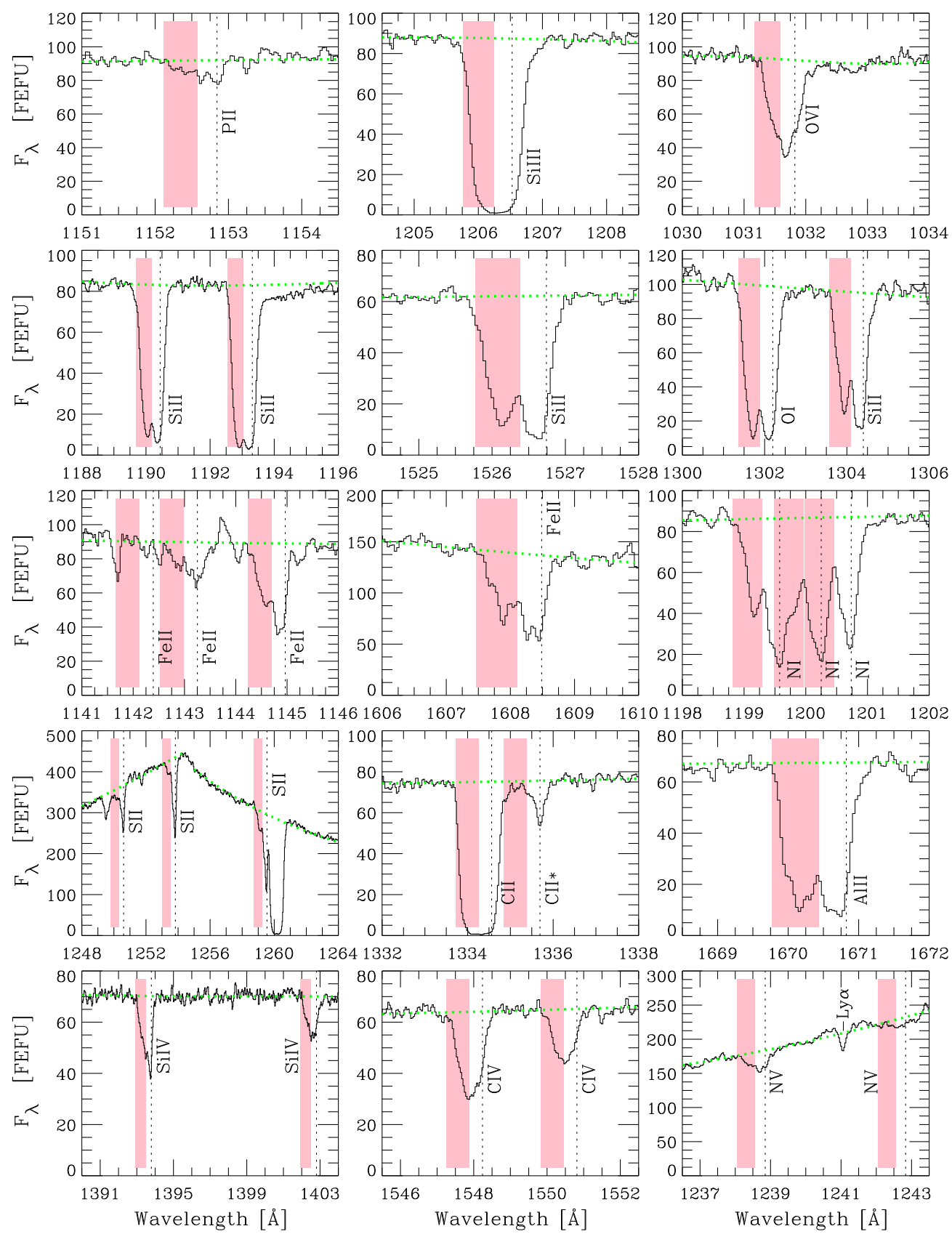

Figure 4. Detail of COS (G130M, G160M) data on HVC at $\left\langle V_{\mathrm{LSR}}\right\rangle=-109 \mathrm{~km} \mathrm{~s}^{-1}$ (range -190 to $-70 \mathrm{~km} \mathrm{~s}^{-1}$ ) toward Mrk 817, showing absorption lines of both low and high ions. HVC Complex $\mathrm{C}$ is seen in UV absorption indicated by pink wash. Fluxes are shown in femto-erg flux units (FEFU) or $10^{-15} \mathrm{erg} \mathrm{cm}^{-2} \mathrm{~s}^{-1} \AA^{-1}$. (A color version of this figure is available in the online journal.)

H I emission, with the exception of the Mrk 876 sight line. In that case, we integrate the UV absorption in Complex C over the range $V_{\mathrm{LSR}}=-170 \mathrm{~km} \mathrm{~s}^{-1}$ to $-100 \mathrm{~km} \mathrm{~s}^{-1}$, whereas the H I emission appears to have a dip at $\left\langle V_{\mathrm{LSR}}\right\rangle=-160 \mathrm{~km} \mathrm{~s}^{-1}$, separating Complex $\mathrm{C}$ from higher-velocity absorption centered at $\left\langle V_{\mathrm{LSR}}\right\rangle=-190 \mathrm{~km} \mathrm{~s}^{-1}$ (see Figures 6 and 10).

From the normalized COS absorption-line data, we derive equivalent widths, line widths, and column densities, which are displayed in Tables 2-5. Details on some of the judgements made in determining column densities are given in Section 3.4. Tables 7-10 compare previous measurements of ion column densities, $\log N\left(X_{i}\right)$, together with our adopted values. We also show the ion ratios referenced to $\log N_{\mathrm{HI}}$ and the corresponding elemental abundances.

\subsection{HVC Column Densities and Abundances}

Tables 7-10 list the adopted and abundances, relative to solar values taken from a recent review (Asplund et al. 2009). On a logarithmic scale where hydrogen is 12.00 , these solar values are C (8.43), N (7.83), O (8.69), S (7.12), Si (7.51), Fe (7.50), $\mathrm{Al}$ (6.45), and $\mathrm{P}(5.41)$. The previous Complex $\mathrm{C}$ abundance estimates were derived from $[\mathrm{O}$ I/ $/ \mathrm{HI}]$ and $[\mathrm{S}$ II/ $\mathrm{HI}]$. The O I measurements should provide an accurate determination of $[\mathrm{O} / \mathrm{H}]$, owing to the strong resonant charge-exchange coupling of $\mathrm{O} I$ and $\mathrm{O}$ II with HII and HI. However, in many sight lines, the OI line ( $\lambda 1302.16)$ is saturated, and we adopted previous values from weaker lines in the FUSE band. Abundances from other ions such as $\left[\mathrm{S}_{\mathrm{II}} / \mathrm{HI}_{\mathrm{I}}\right],\left[\mathrm{Si} \mathrm{II}_{\mathrm{I}} / \mathrm{HI}_{\mathrm{I}}\right]$, and $\left[\mathrm{Fe}_{\mathrm{II}} / \mathrm{HI}_{\mathrm{I}}\right]$ 

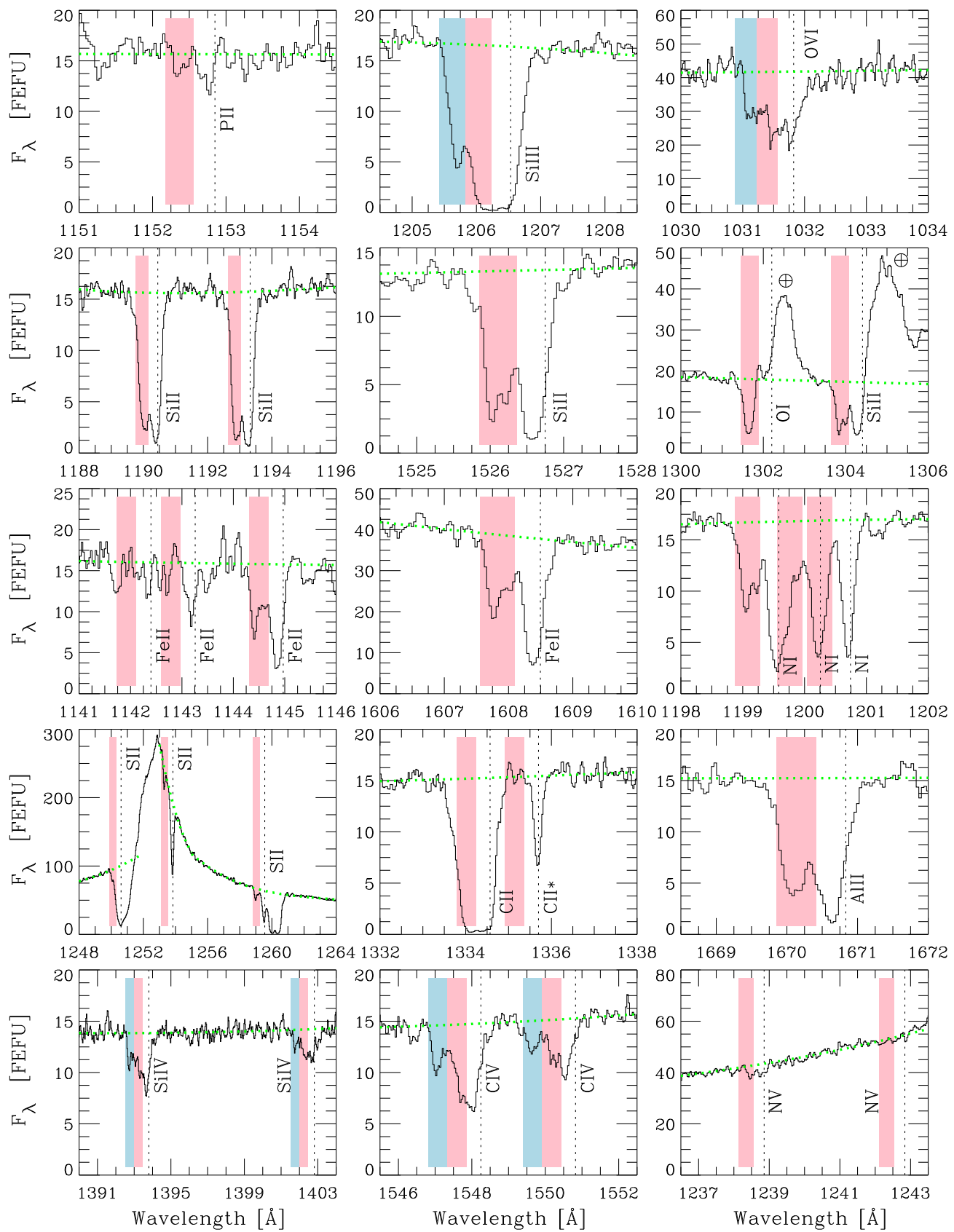

Figure 5. Same as Figure 4 for the Mrk 290 sight line. Note the two HVCs shown by pink and blue wash, corresponding to components at $\left\langle V_{\mathrm{LSR}}\right\rangle=-120 \mathrm{~km} \mathrm{~s}{ }^{-1}$ (range -175 to $-75 \mathrm{~km} \mathrm{~s}^{-1}$ ) and $\left\langle V_{\mathrm{LSR}}\right\rangle=-220 \mathrm{~km} \mathrm{~s}^{-1}$ (range -275 to $-175 \mathrm{~km} \mathrm{~s}^{-1}$ ).

(A color version of this figure is available in the online journal.)

require ionization corrections to arrive at the true metallicities of these elements. There is no guarantee that all elements have the same metallicity, owing to the chemical history of the $\mathrm{HVC}$ or possible dust depletion of refractory elements $(\mathrm{Si}$, $\mathrm{Al}, \mathrm{Fe}$ ). As shown in our previous work (see Figure 16 of CSG03), these corrections are typically expressed as the logarithmic difference between the ion abundance and elemental metallicity. For example, the difference $[\mathrm{S}$ II $/ \mathrm{HI}]-[\mathrm{S} / \mathrm{H}]$ ranges from 0.1 to 0.6 for $\mathrm{HI}$ column densities $\log N_{\mathrm{HI}_{\mathrm{I}}}=$ 19.4-20.1 and HVC physical densities $n_{\mathrm{H}}=0.01-0.1 \mathrm{~cm}^{-3}$. The HVC metallicities are usually found from $\left[\mathrm{O} I / \mathrm{HI}_{\mathrm{I}}\right]$, which requires no ionization correction (CSG03; CSG07), and from [S II/HI], reduced by a factor that depends on the H I column density.
Our four AGN sight lines fall into two distinct groups: one with low column density (Mrk 876 and Mrk 817 have $\left.\log N_{\mathrm{HI}} \approx 19.4-19.5\right)$ and one with high column density (Mrk 290 and PG $1259+593$ have $\log N_{\mathrm{HI}} \approx 20.0-20.1$ ). The ionization corrections to the metal ions (CSG03) depend on the "photoionization parameter," the ratio of ionizing radiation field to gas density $n_{\mathrm{H}}$. As seen in Figure 1, the HVC clumps have characteristic angular sizes of $1^{\circ}-2^{\circ}$, perhaps poorly characterized owing to the 0.6 beam and 0.5 sampling grid of the LAB survey. At the $10 \mathrm{kpc}$ distance of Complex C, $1^{\circ}$ corresponds to $175 \mathrm{pc}$. If the $\mathrm{HI}$ absorbers have a comparable depth and angular extent, the observed H I column densities correspond to physical densities $n_{\mathrm{H}} \approx 0.05 \mathrm{~cm}^{-3}$ (Mrk 876 and Mrk 817) and $n_{\mathrm{H}} \approx 0.2 \mathrm{~cm}^{-3}$ (Mrk 290 and PG 1259). From 

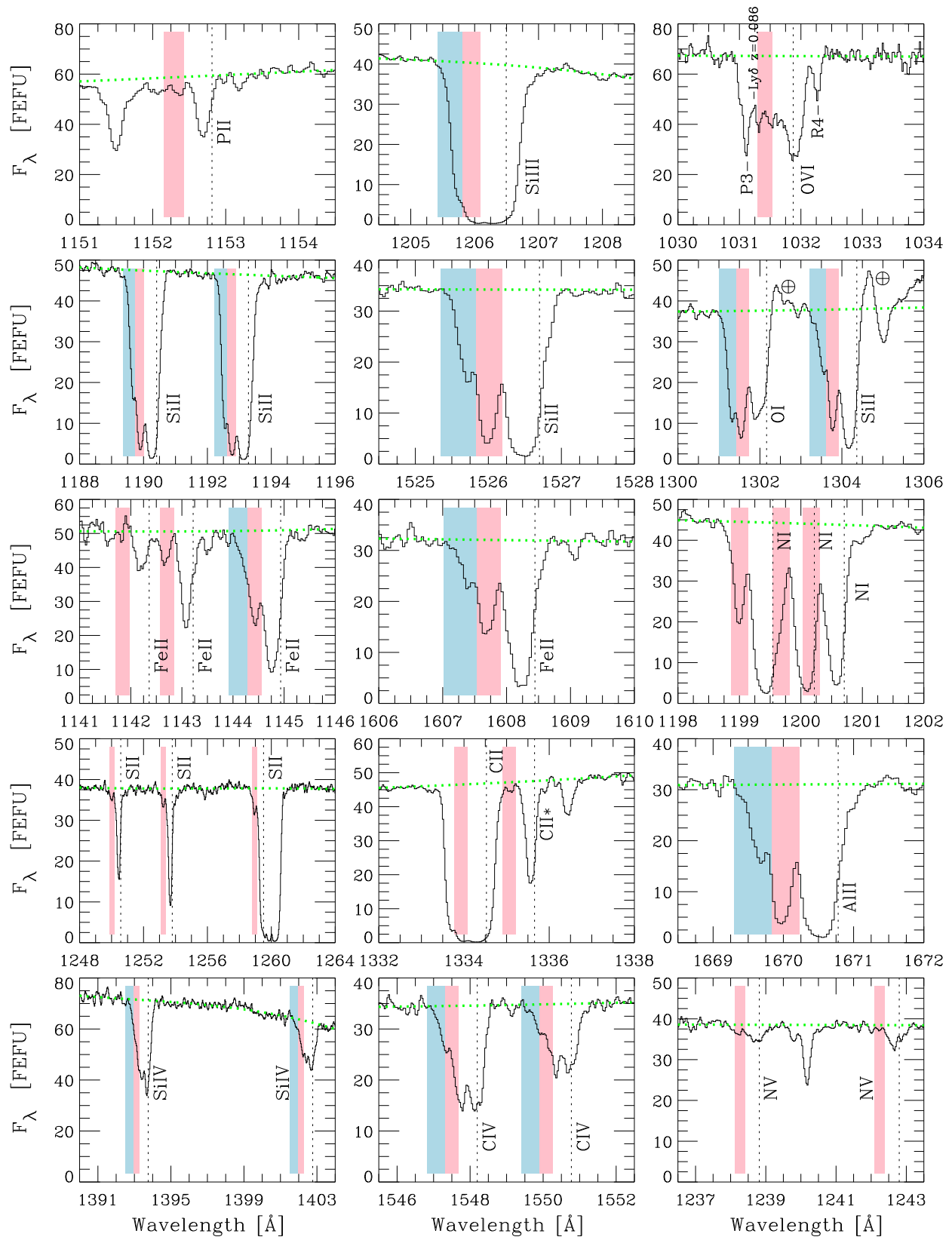

Figure 6. Same as Figure 4 for the Mrk 876 sight line. Note two HVCs shown by pink and blue wash, corresponding to components at $\left\langle V_{\mathrm{LSR}}\right\rangle=-133 \mathrm{~km} \mathrm{~s}{ }^{-1}$ (range -170 to $-100 \mathrm{~km} \mathrm{~s}^{-1}$ ) and $\left\langle V_{\mathrm{LSR}}\right\rangle=-190 \mathrm{~km} \mathrm{~s}^{-1}$ (range -265 to $-160 \mathrm{~km} \mathrm{~s}^{-1}$ ).

(A color version of this figure is available in the online journal.)

our previous photoionization modeling and observed $\mathrm{H}$ I column densities, we adopt the following corrections. For Mrk 290 and PG 1259, the corrections are minor: [S II/HI] and [Si II/H I] are reduced by $0.04(\mathrm{dex})$ and $[\mathrm{Fe} \mathrm{II} / \mathrm{HI}]$ is reduced by 0.02 (dex). For Mrk 876, we reduce [S II/H I] by 0.32 , [Si II/H I] by 0.27 , and $[\mathrm{Fe} \mathrm{II} / \mathrm{HI}$ ] by 0.14 . For Mrk 817 , we reduce [S II/H I] by 0.27 , [Si II/HI] by 0.21 , and $[\mathrm{Fe} \mathrm{II} / \mathrm{HI}]$ by 0.11 . We have not made ionization corrections for O I, N I, Al II, or P II. The ionization corrections noted above for S II, Si II, and Fe II have been applied and included in the final column of Tables 7-10.

\subsection{Inferred Column Densities of Photoionized and Hot Gas}

The Si III column density can be used as a proxy for ionized gas to infer the column density, $N(\mathrm{H}$ II), of ionized hydrogen that is kinematically associated with the observed HI. As discussed by Shull et al. (2009), the Si III ion likely includes contributions from both photoionized and collisionally ionized gas (only $16.34 \mathrm{eV}$ needed to produce $\mathrm{Si}$ III). We adopt an ionization fraction $f_{\mathrm{Si} \text { III }}=0.7 \pm 0.2$ characteristic of multiphase conditions. Following the methodology of our Si III survey with $H S T /$ STIS (Shull et al. 2009), we assume a metallicity $Z_{\mathrm{Si}} \approx 0.1$ relative to the solar abundance, $(\mathrm{Si} / \mathrm{H})_{\odot}=3.24 \times 10^{-5}$, to find

$$
N_{\mathrm{H} \text { II }}=\left(4.4 \times 10^{5}\right) N_{\mathrm{Si} \mathrm{IIII}}\left[\frac{Z_{\mathrm{Si}}}{0.1}\right]^{-1}\left[\frac{f_{\mathrm{Si} \text { IIII }}}{0.7}\right]^{-1} .
$$

For the current four sight lines, we infer ionized column densities, $\log N\left(\mathrm{H}_{\text {II }}\right)=18.79$ (Mrk 290), 19.24 (PG 1259+593), 19.38 (Mrk 817), and 19.56 (Mrk 876). Compared with the observed $\mathrm{HI}$, these correspond to ionization fractions, $N(\mathrm{H}$ II $) /$ $N(\mathrm{H} \mathrm{I})$, ranging from low values of 6\%-10\% in the high-column HVCs toward Mrk $290\left(\log N_{\mathrm{H}_{\mathrm{I}}}=20.05\right)$ and PG $1259+593$ 

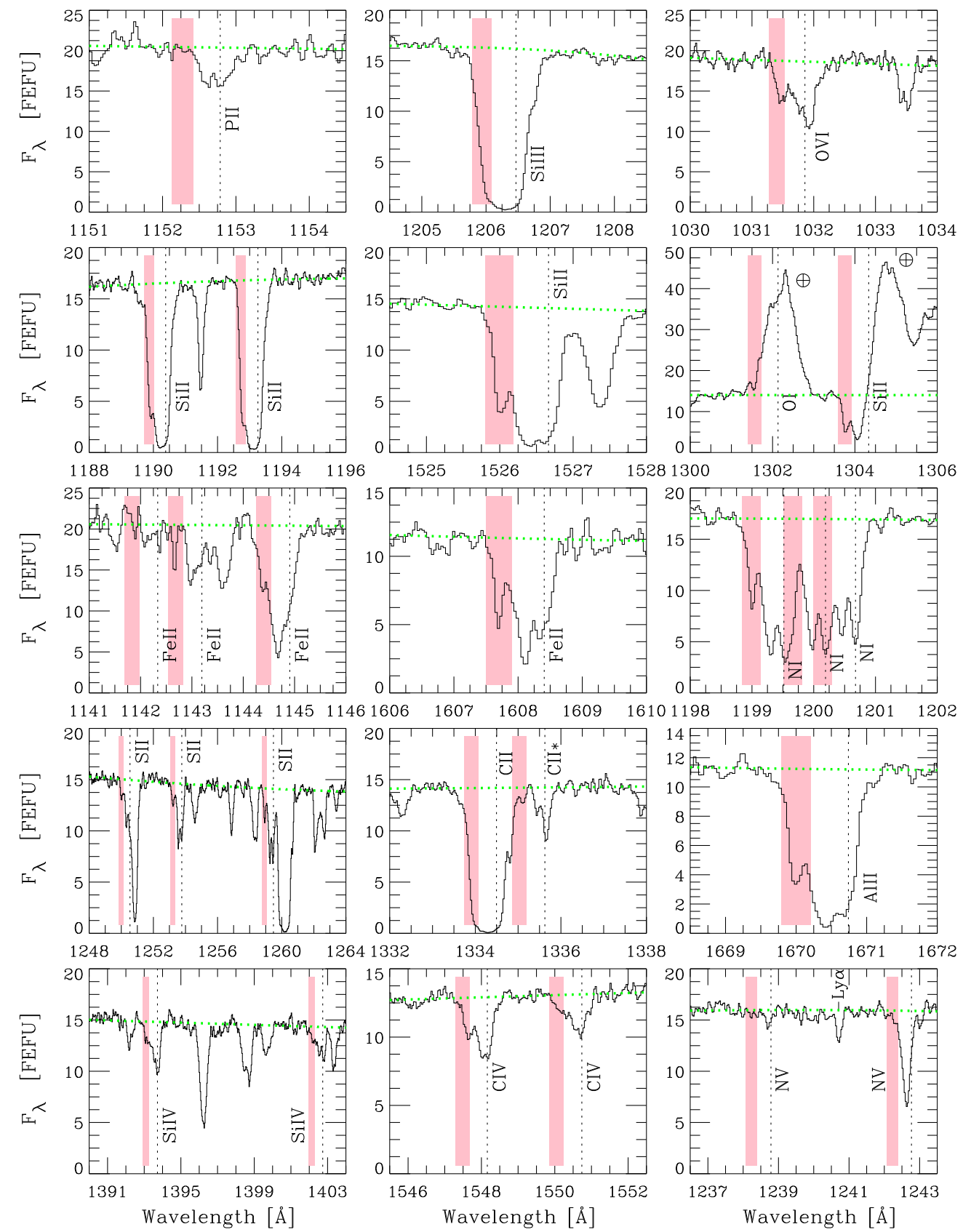

Figure 7. Same as Figure 4 for the PG $1259+593$ sight line. Complex C (pink wash) is seen at $\left\langle V_{\mathrm{LSR}}\right\rangle=-128 \mathrm{~km} \mathrm{~s}^{-1}\left(\mathrm{range}^{-170}\right.$ to $\left.-95 \mathrm{~km} \mathrm{~s}^{-1}\right)$.

(A color version of this figure is available in the online journal.)

$\left(\log N_{\mathrm{HI}}=19.97\right)$ to much larger values of $80 \%$ and $150 \%$ toward Mrk $817\left(\log N_{\mathrm{HI}}=19.50\right)$ and Mrk $876\left(\log N_{\mathrm{HI}}=\right.$ 19.39). Interestingly, the highest ionization fractions occur in the HVCs with the lowest H I column densities. This suggests that the total hydrogen column density distribution is smoother than indicated by the $21 \mathrm{~cm}$ emission maps.

Using arguments similar to the Si III case, we can use the observed column densities of high ions (e.g., $\mathrm{C}$ IV and $\mathrm{O}$ VI) to infer the column densities of hot, collisionally ionized gas. The $\mathrm{COS}$ data on high ions (N v, C IV, O vi, Si IV, Si III) are new or improved over earlier measurements. Our re-measurements of O VI HVC absorption are compared in Section 3.4 to previous FUSE studies (Sembach et al. 2003; Fox et al. 2004; CSG07). In many cases, the COS data have higher $\mathrm{S} / \mathrm{N}$, which we use to define the velocity range of HVC absorption. We re- measured O vi column densities using these ranges (see captions to Figures 4-7). The small differences with previous values (Sembach et al. 2003; Fox et al. 2004) are generally within the stated error bars. We adopt metallicities $Z_{\mathrm{C}}$ and $Z_{\mathrm{O}}$ of $10 \%$ solar and assume hot-gas ionization fractions $f_{\mathrm{C}_{\mathrm{IV}}}=0.3$ and $f_{\mathrm{O} \text { vI }}=0.2$. The observed $\mathrm{C}$ IV and $\mathrm{O}$ VI column densities toward three sight lines (Mrk 817, Mrk 290, Mrk 876) yield consistent "hot gas" column densities $\log N\left(\mathrm{H}_{\mathrm{II}}\right)=18.7-18.8$, with $\mathrm{C}$ IV/O vi abundance ratios consistent, at $N(\mathrm{C}$ IV $) / N(\mathrm{O}$ vI $)=$ $0.4-0.5$. The fourth sight line (PG 1259+593) has the lowest $\mathrm{C}$ IV and $\mathrm{O}$ VI column densities, resulting in a lower inferred $\log N\left(\mathrm{H}_{\mathrm{II}}\right)=18.2-18.3$ and a slightly lower ratio, $N(\mathrm{C}$ IV $) /$ $N(\mathrm{O}$ VI $)=0.3$.

The ionization ratios, $\mathrm{C}$ IV/O vi, Nv/O vi, and Si IV/O vi, can be compared with observations of highly ionized HVCs 


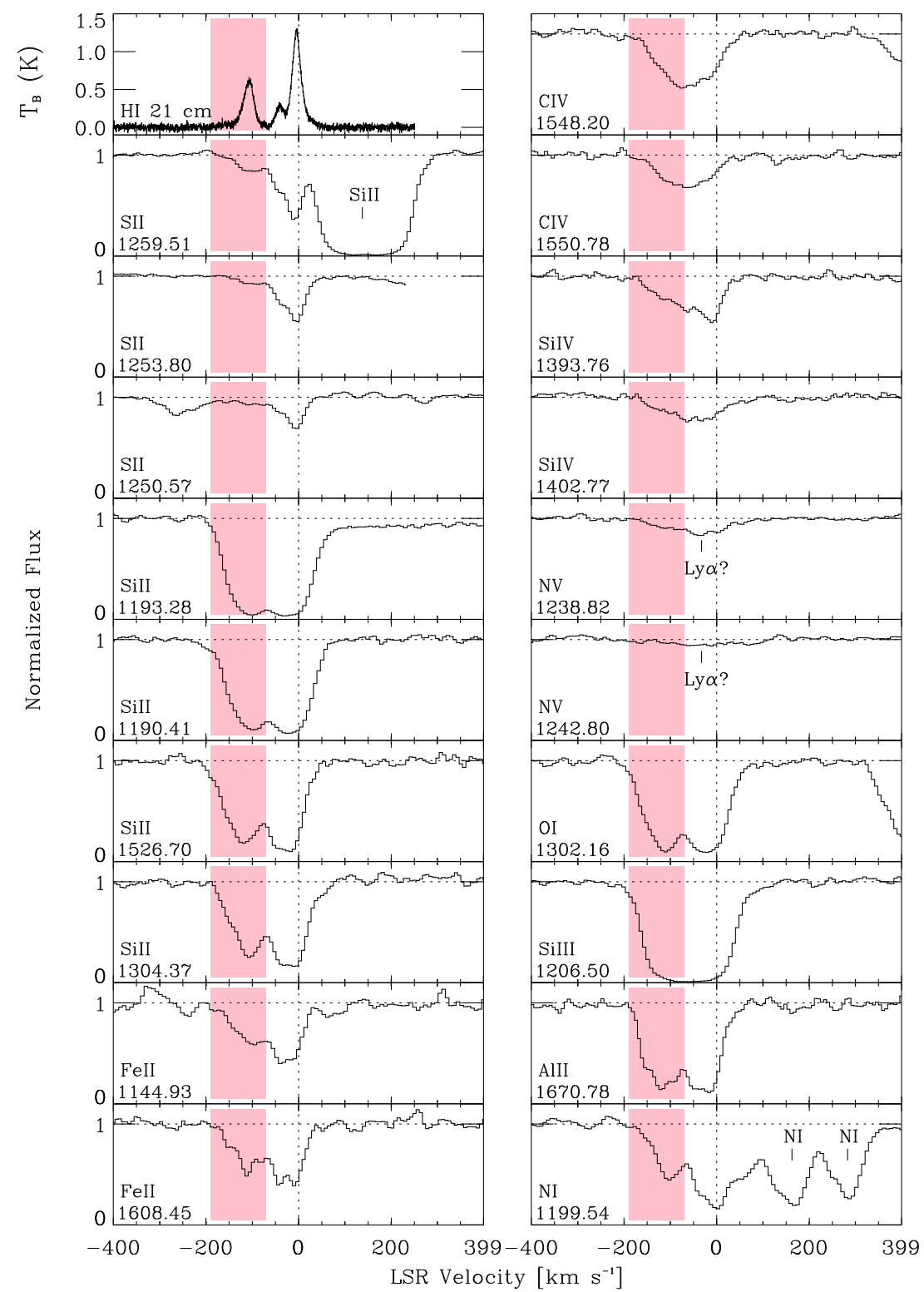

Figure 8. Plot of H I emission (top left panel) and UV ion absorption profiles, continuum-normalized, stacked, and aligned in velocity space for the HVC toward Mrk 817. Complex C (pink wash) appears between $V_{\mathrm{LSR}}=-190$ and $-70 \mathrm{~km} \mathrm{~s}^{-1}$. IGM absorbers (Ly $\alpha$ ) appear to contaminate both N v lines, which we treat as upper limits.

(A color version of this figure is available in the online journal.)

(Fox et al. 2004, 2005, 2006; CSG05; CSG07; Indebetouw \& Shull 2004b) and with models of various ionization processes (see Figure 1 of Indebetouw \& Shull 2004a). In ratio plots of $\mathrm{CIV} / \mathrm{O}$ VI and $\mathrm{NV} / \mathrm{O}$ VI, the regions occupied by HVCs along our four sight lines are consistent with hot gas in conductive or radiatively cooling interfaces. The observed ratios are not consistent with collisional ionization equilibrium (CIE) or turbulent mixing layers (TMLs). For relative solar abundances (O:C:N = 1.00:0.55:0.14), the CIE models produce much higher $\mathrm{Nv} / \mathrm{C}$ IV ratios than observed; the obvious explanation is the lower nitrogen abundance observed in neutral HVC gas. Models of TMLs exhibit a wide range of predictions, owing to the assumptions and parameterizations that go into calculating the ionization and cooling. Early TML models (Slavin et al. 1993; Indebetouw \& Shull 2004a) assumed mixing to an intermediate temperature, $\bar{T} \approx 10^{5} \mathrm{~K}$, and relaxed to ionization equilibrium (Slavin et al. 1993; Indebetouw \& Shull 2004a). Those mod- els produce much higher $\mathrm{C}$ IV $/ \mathrm{O}$ vi ratios than observed. More recent TML models (Kwak \& Shelton 2010) that incorporate non-equilibrium ionization find regions with warm, radiatively cooled C IV, mixed with hotter gas, out of ionization equilibrium. These models find two to four times higher column densities in $\mathrm{N} \mathrm{v}, \mathrm{C}$ IV, and $\mathrm{O}$ VI abundances than predicted in CIE. However, their typical TML ratios are $\mathrm{C} I \mathrm{~V} / \mathrm{O} \mathrm{VI}=1.5$ (range $0.8-2.4$ ) and $\mathrm{Nv} / \mathrm{O}$ vi (range $0.14-0.32$ ), both higher than the COS observations, which have more O vi than predicted by the models. Models of non-equilibrium ionization (Gnat \& Sternberg 2007) with time-dependent cooling $\left(Z \approx 0.1 Z_{\odot}\right)$ find fair agreement with the COS-observed ionization ratios.

We observe a range of ratios, Si IV/C IV $\approx 0.15-0.36$, along the four sight lines, typical of the previous studies (Fox et al. 2004, 2005; CSG05; CSG07). For relative solar abundances, $(\mathrm{Si} / \mathrm{C})_{\odot}=0.11$, photoionization models of HVCs find abundance ratios, $N(\mathrm{Si}$ IV $) / N(\mathrm{C}$ IV $)>1$, for values of photoionization 


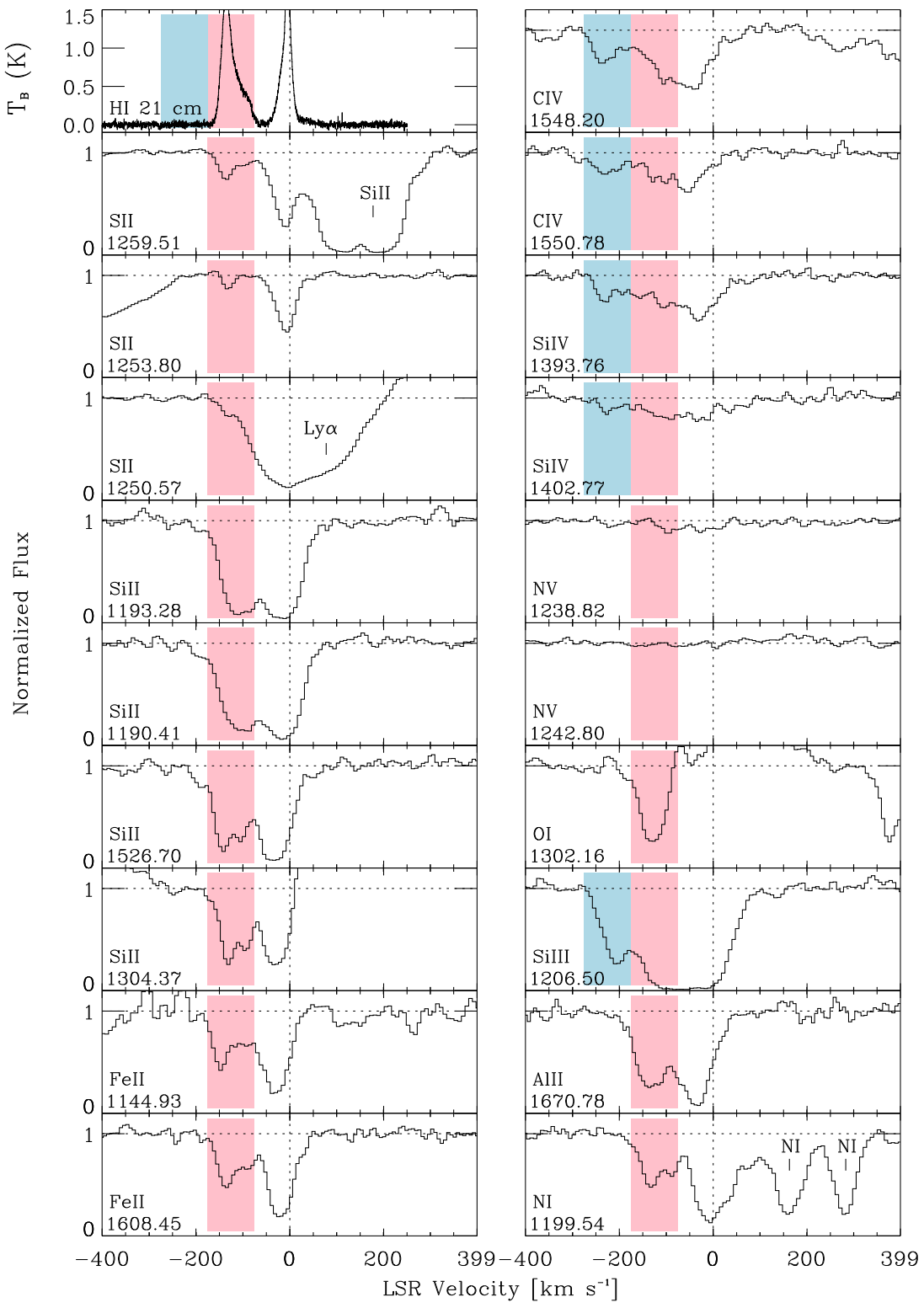

Figure 9. Same as Figure 8 for two HVCs toward Mrk 290, at $\left\langle V_{\mathrm{LSR}}\right\rangle=-120 \mathrm{~km} \mathrm{~s}^{-1}$ (pink wash) and $-220 \mathrm{~km} \mathrm{~s}^{-1}$ (blue wash). Complex C lies at $V_{\mathrm{LSR}}=-160$ to $-75 \mathrm{~km} \mathrm{~s}^{-1}$. An IGM absorber (Ly $\left.\alpha\right)$ is noted in the red wing of $S$ II $\lambda 1250$.

(A color version of this figure is available in the online journal.)

parameter, $\log U=-3.0 \pm 0.2$, needed to explain the low ions and fit the ratios Si IV/Si III/Si II (Shull et al. 2009). These same models also underpredict the total column densities of high ions (C IV, Si IV, N v, O vi). Similarly, photoionization models with the observed range, $N(\mathrm{Si}$ IV $) / N(\mathrm{C}$ IV $)=0.1-0.3$, predict $N(\mathrm{Si}$ IV $) / N(\mathrm{Si}$ II $) \approx 1$, much higher than observed. Therefore, it is likely that some C IV and Si IV comes from hot gas. The kinematic association of low and high ions in these HVCs requires a mixture of denser cloud cores of $\mathrm{H}$ I with extended warm photoionized gas and sheaths of much hotter gas, perhaps produced by bow shocks and turbulent or conductive interfaces between the HVC core and hot halo gas. However, detailed comparisons of observations and models are often complicated by the assumptions of relative solar abundances.

\subsection{Toy Model for HVC Clouds in the Galactic Halo}

One of the reasons for renewed interest in Galactic HVCs is the possibility that the Local Group might contain considerable mass in virialized halos and a hot circumgalactic medium. Spitzer (1956) first suggested the existence of low-density coronal gas with $T=10^{6} \mathrm{~K}$ and $n_{e}=5 \times 10^{-4} \mathrm{~cm}^{-3}$, extending $8 \mathrm{kpc}$ above the Galactic plane and providing pressure confinement of observed high-latitude clouds. Kahn \& Woltjer (1959) noted inconsistencies in Galactic stellar masses and Local Group dynamics (before the inference of dark matter) and suggested the existence of a substantial reservoir of lowdensity halo gas, with $T=5 \times 10^{6} \mathrm{~K}$ and $n_{e}=1 \times 10^{-4} \mathrm{~cm}^{-3}$. Direct probes of hot, low-density gas are difficult, owing to the $n^{2}$ dependence of its X-ray emission and the contamination of most signals by foreground electrons in the kpc-scale "Reynolds layer" (Reynolds 1991). Indirect probes of the halo density yield limits $\left(n_{e}<10^{-4} \mathrm{~cm}^{-3}\right)$ from the effects of drag on orbits of the Magellanic Stream (Moore \& Davis 1994). Similar limits $\left(n_{e}<3 \times 10^{-5} \mathrm{~cm}^{-3}\right)$ follow from ram-pressure stripping of Local Group dwarf galaxies (Blitz \& Robishaw 2000). More recently, Heitsch \& Putman (2009) used numerical 


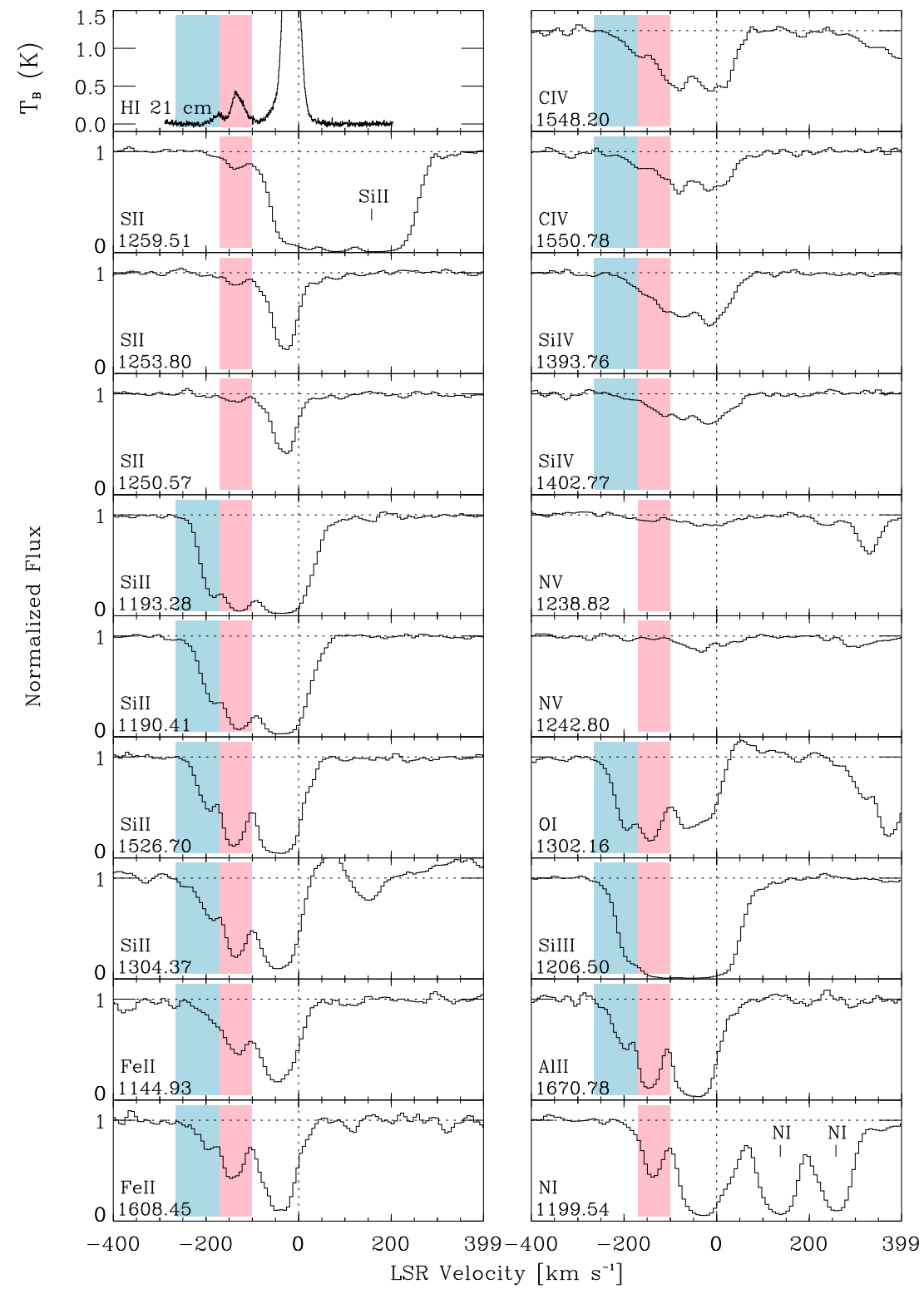

Figure 10. Same as Figure 8 for HVC toward Mrk 876. We find two HVCs at $\left\langle V_{\mathrm{LSR}}\right\rangle=-133 \mathrm{~km} \mathrm{~s}^{-1}$ (pink wash) and $\left\langle V_{\mathrm{LSR}}\right\rangle=-190 \mathrm{~km} \mathrm{~s}{ }^{-1}$ (blue wash).

(A color version of this figure is available in the online journal.)

simulations to suggest that infalling HVCs with $\mathrm{H}$ I masses less than $10^{4.5} M_{\odot}$ may become fully ionized by Kelvin-Helmholtz instabilities within $10^{8} \mathrm{yr}(\sim 10 \mathrm{kpc}$ for typical HVC velocities of $100 \mathrm{~km} \mathrm{~s}^{-1}$ ). All of these estimates depend critically on the assumed halo gas density and on dynamical interactions at the boundaries between the HVCs and the hot, low-density medium that confines them.

As a toy model for HVC cloud confinement, we consider spherical clouds with radius $R$, mass $M$, constant gas density $\rho$, and mean atomic mass $\mu=\rho / n_{\mathrm{H}}=1.23 m_{\mathrm{H}}$ (helium $25 \%$ by mass) in virial equilibrium confined by external pressure, $P_{0}$. For clumps of angular radius $\theta_{\mathrm{deg}}$ (in degrees), we adopt $R=(175 \mathrm{pc}) \theta_{\mathrm{deg}}$ at $10 \mathrm{kpc}$ distance, $n_{\mathrm{H}}=\left(0.1 \mathrm{~cm}^{-3}\right) n_{0.1}$, and temperature $T=(100 \mathrm{~K}) T_{100}$. The virial theorem with confinement (Spitzer 1978) requires that

$$
4 \pi R^{3} P_{0}=\frac{3 M k T}{\mu}-\frac{3 G M^{2}}{5 R} .
$$

The confining pressure could arise from hot Galactic halo gas, which we scale to nominal values $n_{\text {halo }}=\left(10^{-5} \mathrm{~cm}^{-3}\right) n_{-5}$ and $T_{\text {halo }}=\left(10^{6} \mathrm{~K}\right) T_{6}$. Alternately, the HVCs could be confined by ram pressure, $P_{\text {ram }}=\rho_{\text {halo }} V_{\mathrm{HVC}}^{2}$, as they fall through the halo. For the assumed halo parameters, the total thermal pressure of fully ionized gas with $n_{\mathrm{He}} / n_{\mathrm{H}}=0.0823$ is $P / k \approx$ $2.25 n_{\mathrm{H}} T=\left(22.5 \mathrm{~cm}^{-3} \mathrm{~K}\right) n_{-5} T_{6}$. This pressure is consistent with inferences from various highly ionized HVCs (Sembach et al. 1999; CSG05), although such estimates are uncertain owing to assumptions in the ionization modeling. The halo density probably lies in the range $n_{e}=(1-10) \times 10^{-5} \mathrm{~cm}^{-3}$, with considerable variation over vertical distances $5-50 \mathrm{kpc}$ above the Galactic plane.

In our model, the HVC clump masses in Complex $\mathrm{C}$ are $M=\left(6.8 \times 10^{4} M_{\odot}\right) n_{0.1} \theta_{\mathrm{deg}}^{3}$, and the terms in the virial equation are all of comparable size,

$$
(3 M k T / \mu)=\left(2.7 \times 10^{48} \mathrm{erg}\right) n_{0.1} T_{100} \theta_{\mathrm{deg}}^{3}
$$




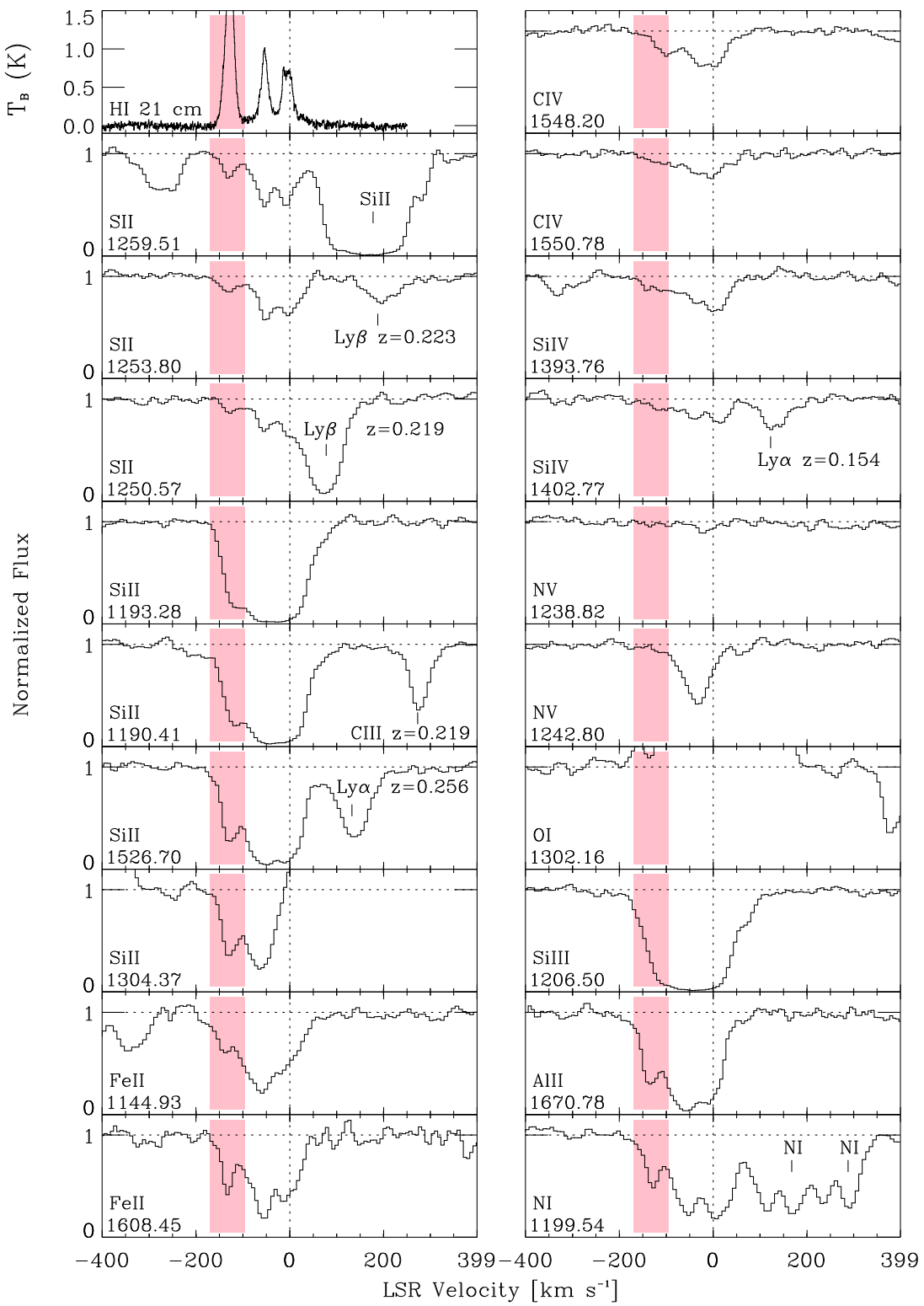

Figure 11. Same as Figure 8 for HVC at $\left\langle V_{\mathrm{LSR}}\right\rangle=-128 \mathrm{~km} \mathrm{~s}^{-1}$ (pink wash) toward PG 1259+593. IGM absorbers (Ly $\alpha$, Ly $\beta, \mathrm{C}$ III) are noted. (A color version of this figure is available in the online journal.)

$$
\begin{gathered}
\left(3 G M^{2} / 5 R\right)=\left(1.4 \times 10^{48} \mathrm{erg}\right) n_{0.1}^{2} \theta_{\mathrm{deg}}^{5} \\
4 \pi R^{3} P_{0}=\left(4.0 \times 10^{48} \mathrm{erg}\right)\left[n_{\text {halo }} / 10^{-5} \mathrm{~cm}^{-3}\right] \theta_{\mathrm{deg}}^{3}
\end{gathered}
$$

The thermal pressure of the halo, $P_{\text {halo }} \approx 1.6 \times 10^{-15} \mathrm{erg} \mathrm{cm}^{-3}$, is comparable to the ram pressure on the HVCs. This not surprising, as these infalling clouds have low Mach numbers with respect to the hot gas. The HVC clumps may not be selfgravitating (no $\mathrm{H}_{2}$ or stars have been detected). However, their observed properties place them near virial equilibrium (with $T \leqslant 10^{3} \mathrm{~K}$ ), and there may be no need to invoke dark matter for their confinement.

As the HVCs encounter the higher densities in the stratified lower halo, their outer portions may be torn apart and dissipated by interface instabilities. This would feed the Galactic halo rather than replenishing the reservoir of star formation in the disk. Portions of Complex $\mathrm{C}$ do appear to be clumping up, although the individual clump masses $\left(\theta_{\mathrm{deg}} \geqslant 1\right)$ are likely above the $10^{4.5} M_{\odot}$ threshold for survival (Heitsch \& Putman 2009).

\subsection{Notes on Individual Sight Lines}

In this section, we compare the COS equivalent widths of several lines, previously measured by GHRS and STIS spectrographs (CSG03; CSG07; Richter et al. 2001). The derived column densities of various ions are compared in Tables 7-10. For some of the high ions (CIV, Si IV, Si III, $\mathrm{Nv}$ ) our COS measurements are the only published data. Previous measurements of high-velocity Si III toward Mrk 876 and PG $1259+593$ were discussed in the HST surveys by Shull et al. (2009) and Collins et al. (2009).

1. Mrk 817. The three unsaturated S II lines at 1250, 1253, and $1259 \AA$ should have equivalent widths in the ratio 1:2:3 based on their relative oscillator strengths. The weakest line $(\lambda 1250.6)$ was measured at $W_{\lambda}=27 \pm 8 \mathrm{~m} \AA$ (COS) versus $16 \pm 3 \mathrm{~m} \AA$ (GHRS). The stronger line $(\lambda 1253.8)$ 
Table 7

Mrk 817: Summary of Column Densities ${ }^{\mathrm{a}}$ and Abundances

\begin{tabular}{|c|c|c|c|c|c|}
\hline $\begin{array}{l}\text { Species } \\
\left(X_{i}\right)\end{array}$ & $\begin{array}{l}\text { CSG-03 } \\
\left(\log N_{X_{i}}\right)\end{array}$ & $\begin{array}{l}\text { Shull-11 } \\
\left(\log N_{X_{i}}\right)\end{array}$ & $\begin{array}{l}\text { Adopted } \\
\left(\log N_{X_{i}}\right)\end{array}$ & $\begin{array}{c}\text { Abundance } \\
\log \left(N_{X_{i}} / N_{\mathrm{H}_{\mathrm{I}}}\right)\end{array}$ & $\begin{array}{c}\text { Abundance }^{\mathrm{b}} \\
{[X / H]}\end{array}$ \\
\hline O I & $15.72_{-0.16}^{+0.24}$ & $\geqslant 14.98$ & $15.72_{-0.16}^{+0.24}$ & $-3.78 \pm 0.24$ & $-0.47 \pm 0.24$ \\
\hline $\mathrm{N}_{\mathrm{I}}$ & $<14.05$ & $14.06_{-0.07}^{+0.12}$ & $14.06_{-0.07}^{+0.12}$ & $-5.44 \pm 0.10$ & $-1.27 \pm 0.10$ \\
\hline S II & $14.34_{-0.05}^{+0.05}$ & $14.29_{-0.08}^{+0.08}$ & $14.29_{-0.08}^{+0.08}$ & $-5.21 \pm 0.08$ & $-0.60 \pm 0.10$ \\
\hline $\mathrm{Si}$ II & $14.48_{-0.08}^{+0.07}$ & $14.47_{-0.05}^{+0.08}$ & $14.47_{-0.05}^{+0.08}$ & $-5.03 \pm 0.08$ & $-0.75 \pm 0.10$ \\
\hline Fe II & $14.31_{-0.08}^{+0.11}$ & $14.23_{-0.06}^{+0.10}$ & $14.23_{-0.06}^{+0.10}$ & $-5.27 \pm 0.10$ & $-0.88 \pm 0.15$ \\
\hline $\mathrm{C}_{\text {II }}$ & $\ldots$ & $\geqslant 14.9$ & $\geqslant 14.9$ & $>-4.6$ & $\geqslant-1.03$ \\
\hline Al II & $\ldots$ & $13.23_{-0.06}^{+0.07}$ & $13.23_{-0.06}^{+0.07}$ & $-6.27 \pm 0.07$ & $-0.72 \pm 0.07$ \\
\hline $\mathrm{P}_{\mathrm{II}}$ & & $<12.95$ & $<12.95$ & $<-6.55$ & $<0.04$ \\
\hline C IV & $\ldots$ & $13.77_{-0.09}^{+0.14}$ & $13.77_{-0.09}^{+0.14}$ & $-5.73_{-0.09}^{+0.14}$ & \\
\hline $\mathrm{N} \mathrm{v}$ & $\ldots$ & $13.16_{-0.13}^{+0.10}$ & $13.16_{-0.13}^{+0.10}$ & $-6.34_{-0.13}^{+0.10}$ & \\
\hline O VI & $\ldots$ & $14.05_{-0.09}^{+0.16}$ & $14.05_{-0.09}^{+0.16}$ & $-5.45_{-0.09}^{+0.16}$ & \\
\hline Si IV & $\ldots$ & $13.08_{-0.12}^{+0.13}$ & $13.08_{-0.12}^{+0.13}$ & $-6.42_{-0.12}^{+0.13}$ & \\
\hline Si III & $\ldots$ & $13.74_{-0.13}^{+0.15}$ & $13.74_{-0.13}^{+0.15}$ & $-5.76_{-0.13}^{+0.15}$ & \\
\hline
\end{tabular}

\section{Notes.}

a Comparison of measured column densities, $\log N_{a}\left(\mathrm{~cm}^{-2}\right)$, for $\mathrm{HVC}$ at $\left\langle V_{\mathrm{LSR}}\right\rangle=-109 \mathrm{~km} \mathrm{~s}^{-1}$ (integrated from $-190 \mathrm{~km} \mathrm{~s}^{-1}$ to $-70 \mathrm{~km} \mathrm{~s}^{-1}$ ).

b Abundances for neutrals and first ions $X_{i}$ are given relative to measured $\log N_{\mathrm{H}_{\mathrm{I}}}=19.50 \pm 0.01$ (Table 2). Fox et al. (2004) found $\log N(\mathrm{O} \mathrm{VI})=$ $13.97_{-0.13}^{+0.10}$ for HVC absorption between -160 and $-80 \mathrm{~km} \mathrm{~s}^{-1}$. Last column gives abundances of elements $[X / H]$ relative to solar abundances (Asplund et al. 2009), with ionization corrections of $0.27 \mathrm{dex}$ (S II), $0.21 \mathrm{dex}$ (Si II), and 0.11 dex (Fe II) subtracted from the first-ion abundances (Section 3.2).

was $19 \pm 5 \mathrm{~m} \AA(\mathrm{COS})$ versus $28 \pm 3 \mathrm{~m} \AA$ (GHRS). The strongest line ( $\lambda 1259.5)$ with $42 \pm 7 \mathrm{~m} \AA$ (COS) was not reported with GHRS. We base our S II column density, $\log N=14.29 \pm 0.08$, on the $\lambda 1259$ measurement. For other lines we used Si IV $\lambda 1394$, N v $\lambda 1238$, Si II $\lambda 1304$ (consistent with $\lambda 1526$ ), and Fe II $\lambda 1608$ (consistent with $\lambda 1145)$. We find good agreement between both lines in the doublets of C IV $(\lambda \lambda 1548,1551), \mathrm{N}$ V $(\lambda \lambda 1238,1242)$, and Si IV $(\lambda \lambda 1394,1402)$. Fox et al. (2004) suggested that there may be no detectable high-velocity $\mathrm{N} v$ toward Mrk 817 because of intergalactic $\operatorname{Ly} \alpha$ absorbers at $z=0.0189$ and $z=0.0194$. However, we believe we have detected $\mathrm{N} \mathrm{v}$ with $\log N=13.16_{-0.15}^{+0.12}$ at the $-109 \mathrm{~km} \mathrm{~s}^{-1}$ velocity of Complex C. As seen in Figure 8, the Ly $\alpha$ absorber at $z=0.0184$ would appear at $-42 \mathrm{~km} \mathrm{~s}^{-1}$ in the $\mathrm{Nv}$ rest frame, fairly well separated from the HVC. The ratios of $\mathrm{N} \mathrm{v}$ to other high ions (C IV, Si IV, O VI) are typical of other sight lines. We use the weaker $N_{\text {I }} \lambda 1199.5$ line, since the other lines $(1200.2 \AA$ and $1200.7 \AA)$ are blended with $V_{\text {LSR }}=0$ absorption. Both $\mathrm{Al}$ II $\lambda 1670.78$ and Si III $\lambda 1206.50$ are mildly saturated, at equivalent widths of $\sim 400 \mathrm{~m} \AA$ and $\sim 350 \mathrm{~m} \AA$, respectively. Our inferred column densities from AOD integration of $N_{a}(v)$ accurately reflect this saturation, for line broadening of $20-30 \mathrm{~km} \mathrm{~s}^{-1}$, somewhat higher than the Doppler parameter $b=11 \mathrm{~km} \mathrm{~s}^{-1}$ inferred from $\mathrm{O}$ I and other low ions (CSG03). Re-measuring the $\mathrm{O}$ VI column density, we find $\log N=14.05_{-0.09}^{+0.16}$ compared to $13.97_{-0.13}^{+0.10}$ from Fox et al. (2004) and $13.88 \pm 0.20$ (Sembach et al. 2003). The small differences in O vi column densities arise from the velocity range adopted for the HVC absorption. From the extent of UV absorption (S II, Si II, Fe II, Al II) in Figure 8, we integrate between $V_{\text {LSR }}=-190$ and $-70 \mathrm{~km} \mathrm{~s}^{-1}$, whereas Fox et al. (2004) used the interval from -160 to $-80 \mathrm{~km} \mathrm{~s}^{-1}$.
2. Mrk 290. The S II data from COS are superior to that from GHRS or STIS (CSG03; CSG07). The HVC components of $\lambda 1250.57$ and $\lambda 1253.80$ lie in the blue and red wings, respectively, of the broad Ly $\alpha$ emission line of the AGN (see Figure 5). To arrive at the column density, $\log N=14.43 \pm$ 0.08 , we use the $\lambda 1259.51$ line. For Si II, both lines $(\lambda 1304$ and $\lambda 1526)$ are saturated, yielding a lower limit on column density. We adopt the CoG value, $\log N=14.9 \pm 0.15$, from CSG07. The column density from the mildly saturated Fe II $\lambda 1608$ is consistent with $\lambda 1145$. We see agreement between both lines in the doublets of C IV $(\lambda \lambda 1548,1551)$, $\mathrm{Nv}(\lambda \lambda 1238,1242)$, and Si IV $(\lambda \lambda 1394,1402)$. We use the weaker line $\mathrm{N}_{\mathrm{I}} \lambda 1199.5$ line, since the other lines $(1200.2 \AA$ and $1200.7 \AA)$ are blended with $V_{\mathrm{LSR}}=0$ absorption. Both $\mathrm{Al}$ II $\lambda 1670.78$ and Si III $\lambda 1206.50$ are mildly saturated, at equivalent widths of $\sim 340 \mathrm{~m} \AA$ and $\sim 345 \mathrm{~m} \AA$, respectively. Our inferred column densities from AOD integration of $N_{a}(v)$ accurately reflect this saturation, for line broadening of $20-30 \mathrm{~km} \mathrm{~s}^{-1}$, somewhat higher than the Doppler parameter $b=16 \mathrm{~km} \mathrm{~s}^{-1}$ inferred from $\mathrm{O} \mathrm{I}$ and low ions (CSG07). We re-measured the $\mathrm{O}$ vi column density, finding reasonable agreement, $\log N=14.10_{-0.14}^{+0.15}$ (between -175 and $-75 \mathrm{~km} \mathrm{~s}^{-1}$ ) compared to $14.23 \pm 0.04$ (CSG07 integrated between -165 and $-75 \mathrm{~km} \mathrm{~s}^{-1}$ ) and $14.20 \pm 0.16$ (Sembach et al. 2003).

3. Mrk 876. The S II data from COS are superior to that from STIS (CSG07), which only gave an upper limit, $\log N<14.34$. With COS, the HVC components of $\lambda 1259.51$ and $\lambda 1253.80$ yield essentially the same column density, $\log N=14.25_{-0.14}^{+0.13}$. For Si II, both lines $(\lambda 1304$ and $\lambda 1526)$ are mildly saturated, but yield a consistent column density, $\log N=14.41_{-0.09}^{+0.16}$. The column density from Fe II $\lambda 1608, \log N=14.26_{-0.10}^{+0.17}$, is consistent with that from $\lambda 1145$. We find agreement between both lines in the doublets of C IV $(\lambda \lambda 1548,1551), \mathrm{N} v(\lambda \lambda 1238,1242)$, and Si IV $(\lambda \lambda 1394,1402)$. We use the weaker $N_{\text {I }} \lambda 1199.5$ line, since the other lines (1200.2 $\AA$ and $1200.7 \AA$ ) are blended with $V_{\mathrm{LSR}}=0$ absorption. Both Al II $\lambda 1670.78$ and Si III $\lambda 1206.50$ are mildly saturated, at equivalent widths of $\sim 280 \mathrm{~m} \AA$ and $\sim 270 \mathrm{~m} \AA$, respectively. Our inferred column densities from AOD integration of $N_{a}(v)$ accurately reflect this saturation, for line broadening of $20 \mathrm{~km} \mathrm{~s}^{-1}$, somewhat higher than the Doppler parameter $b=16 \mathrm{~km} \mathrm{~s}^{-1}$ inferred from O I and low ions (CSG03). Our previous HVC survey of Si III (Shull et al. 2009) measured $\log N \geqslant 13.92$ for this absorber, slightly higher than the value, $13.72_{-0.14}^{+0.24}$ measured here. We re-measured the $\mathrm{O}$ vi column density, finding moderate differences, but within stated errors, $\log N=13.99_{-0.14}^{+0.36}$ compared to $14.20 \pm 0.02$ (CSG07), $14.05 \pm 0.17$ (Sembach et al. 2003), and 14.12 ${ }_{-0.13}^{+0.11}$ (Fox et al. 2004). From the observed extent of the UV absorption (S II, Si II, Fe II), we integrate O vi between $V_{\mathrm{LSR}}=-170$ and $-100 \mathrm{~km} \mathrm{~s}^{-1}$, whereas Fox et al. (2004) used the interval from -220 to $-100 \mathrm{~km} \mathrm{~s}^{-1}$, and CSG07 used -210 to $-75 \mathrm{~km} \mathrm{~s}^{-1}$.

4. $P G 1259+593$. The $\mathrm{S}$ II data from $\mathrm{COS}$ are superior to that from GHRS (CSG03) and agree well with those from STIS (Sembach et al. 2004; Richter et al. 2001). Our column density, $\log N=14.35_{-0.10}^{+0.15}$, agrees with previous measurements. For Si II, both lines ( $\lambda 1304$ and $\lambda 1526)$ are mildly saturated and yield a consistent column density. The column density from Fe II $\lambda 1608$ is consistent with $\lambda 1145$. We find agreement between both lines in 
Table 8

Mrk 290: Summary of Column Densities ${ }^{\mathrm{a}}$ and Abundances

\begin{tabular}{|c|c|c|c|c|c|c|}
\hline $\begin{array}{l}\text { Species } \\
\left(X_{i}\right)\end{array}$ & $\begin{array}{c}\text { CSG-03 } \\
\left(\log N_{X_{i}}\right)\end{array}$ & $\begin{array}{c}\text { CSG-07 } \\
\left(\log N_{X_{i}}\right)\end{array}$ & $\begin{array}{c}\text { Shull-11 } \\
\left(\log N_{X_{i}}\right)\end{array}$ & $\begin{array}{c}\text { Adopted } \\
\left(\log N_{X_{i}}\right)\end{array}$ & $\begin{array}{c}\text { Abundance }^{\mathrm{b}} \\
\log \left(N_{X_{i}} / N_{\mathrm{H}_{\mathrm{I}}}\right)\end{array}$ & $\begin{array}{c}\text { Abundance }^{\mathrm{b}} \\
{[X / H]}\end{array}$ \\
\hline O I & $<16.79$ & $15.75_{-0.15}^{+0.29}$ & $14.64_{-0.02}^{+0.03}$ & $15.75_{-0.15}^{+0.29}$ & $-4.30_{-0.15}^{+0.29}$ & $-0.99_{-0.15}^{+0.29}$ \\
\hline $\mathrm{NI}_{\mathrm{I}}$ & $<15.02$ & $14.23_{-0.20}^{+0.17}$ & $14.06_{-0.07}^{+0.12}$ & $14.06_{-0.07}^{+0.12}$ & $-5.99_{-0.07}^{+0.12}$ & $-1.82_{-0.07}^{+0.12}$ \\
\hline$S_{\text {II }}$ & $14.29_{-0.14}^{+0.15}$ & $14.24_{-0.20}^{+0.16}$ & $14.43_{-0.08}^{+0.08}$ & $14.43_{-0.08}^{+0.08}$ & $-5.62_{-0.08}^{+0.08}$ & $-0.78_{-0.15}^{+0.15}$ \\
\hline Si II & $<14.97$ & $14.93_{-0.13}^{+0.18}$ & $>14.40$ & $14.93_{-0.13}^{+0.18}$ & $-5.12_{-0.13}^{+0.18}$ & $-0.67_{-0.15}^{+0.15}$ \\
\hline Fe II & $<15.46$ & $14.41_{-0.21}^{+0.23}$ & $14.26_{-0.05}^{+0.09}$ & $14.26_{-0.05}^{+0.09}$ & $-5.79_{-0.05}^{+0.09}$ & $-1.31_{-0.05}^{+0.09}$ \\
\hline $\mathrm{Al}$ II & $\ldots$ & $\ldots$ & $13.11_{-0.09}^{+0.12}$ & $13.11_{-0.09}^{+0.12}$ & $-6.94_{-0.09}^{+0.12}$ & $-1.39_{-0.09}^{+0.12}$ \\
\hline $\mathrm{P}_{\mathrm{II}}$ & $\ldots$ & $<12.82$ & $12.83_{-0.18}^{+0.22}$ & $12.83_{-0.18}^{+0.22}$ & $-7.22_{-0.18}^{+0.22}$ & $-0.63_{-0.18}^{+0.22}$ \\
\hline CiV & $\ldots$ & $\ldots$ & $13.78_{-0.09}^{+0.14}$ & $13.78_{-0.09}^{+0.14}$ & $-6.27_{-0.09}^{+0.14}$ & \\
\hline $\mathrm{Nv}$ & $\ldots$ & $<13.49$ & $12.98_{-0.15}^{+0.22}$ & $12.98_{-0.15}^{+0.22}$ & $-7.07_{-0.15}^{+0.22}$ & \\
\hline O VI & $\ldots$ & $14.23_{-0.04}^{+0.04}$ & $14.10_{-0.14}^{+0.15}$ & $14.10_{-0.14}^{+0.15}$ & $-5.95_{-0.14}^{+0.15}$ & \\
\hline Si IV & $\ldots$ & $\ldots$ & $12.95_{-0.12}^{+0.12}$ & $12.95_{-0.12}^{+0.12}$ & $-7.10_{-0.12}^{+0.12}$ & \\
\hline Si III & $\ldots$ & $\ldots$ & $13.15_{-0.08}^{+0.10}$ & $13.15_{-0.08}^{+0.10}$ & $-6.90_{-0.08}^{+0.10}$ & \\
\hline
\end{tabular}

Notes.

${ }^{\text {a }}$ Comparison of measured column densities, $\log N_{a}\left(\mathrm{~cm}^{-2}\right)$, for $\mathrm{HVC}$ at $\left\langle V_{\mathrm{LSR}}\right\rangle=-120 \mathrm{~km} \mathrm{~s}^{-1}$ (integrated from $-175 \mathrm{~km} \mathrm{~s}^{-1}$ to $-75 \mathrm{~km} \mathrm{~s}^{-1}$ ).

${ }^{\mathrm{b}}$ Abundances for neutrals and first ions $X_{i}$ are given relative to measured $\log N_{\mathrm{H}_{\mathrm{I}}}=20.05 \pm 0.02$ for all three HVC components (Table 2). Wakker et al. (1999) found $\log N(\mathrm{~S}$ II $)=14.34_{-0.11}^{+0.08}$. The last column gives elemental abundances $[X / H]$ relative to solar values (Asplund et al. 2009) with ionization corrections of 0.04 dex (S II), $0.04 \mathrm{dex}$ (Si II), and $0.02 \mathrm{dex}$ (Fe II) subtracted from the first-ion abundances (Section 3.2).

Table 9

Mrk 876: Summary Column Densities ${ }^{\mathrm{a}}$ and Abundances

\begin{tabular}{|c|c|c|c|c|c|c|}
\hline $\begin{array}{l}\text { Species } \\
\left(X_{i}\right)\end{array}$ & $\begin{array}{c}\text { CSG-03 } \\
\left(\log N_{X_{i}}\right)\end{array}$ & $\begin{array}{c}\text { CSG-07 } \\
\left(\log N_{X_{i}}\right)\end{array}$ & $\begin{array}{c}\text { Shull-11 } \\
\left(\log N_{X_{i}}\right)\end{array}$ & $\begin{array}{c}\text { Adopted } \\
\left(\log N_{X_{i}}\right)\end{array}$ & $\begin{array}{c}\text { Abundance }^{\mathrm{b}} \\
\log \left(N_{X_{i}} / N_{\mathrm{H}_{\mathrm{I}}}\right)\end{array}$ & $\begin{array}{c}\text { Abundance }^{\mathrm{b}} \\
{[X / H]}\end{array}$ \\
\hline OI & $15.55_{-0.28}^{+0.42}$ & $15.26_{-0.14}^{+0.17}$ & $>14.74$ & $15.26_{-0.14}^{+0.17}$ & $-4.13_{-0.14}^{+0.17}$ & $-0.82_{-0.14}^{+0.17}$ \\
\hline NI & $14.20_{-0.14}^{+0.15}$ & $13.90_{-0.04}^{+0.03}$ & $13.97_{-0.08}^{+0.13}$ & $13.97_{-0.08}^{+0.13}$ & $-5.42_{-0.08}^{+0.13}$ & $-1.25_{-0.08}^{+0.13}$ \\
\hline$S_{\text {II }}$ & $\ldots$ & $<14.34$ & $14.25_{-0.14}^{+0.13}$ & $14.25_{-0.14}^{+0.13}$ & $-5.14_{-0.14}^{+0.13}$ & $-0.58_{-0.20}^{+0.20}$ \\
\hline Si II & $14.53_{-0.13}^{+0.11}$ & $14.56_{-0.09}^{+0.11}$ & $14.41_{-0.09}^{+0.16}$ & $14.41_{-0.09}^{+0.16}$ & $-4.98_{-0.14}^{+0.13}$ & $-0.76_{-0.20}^{+0.20}$ \\
\hline $\mathrm{Fe}$ II & $14.41_{-0.08}^{+0.09}$ & $14.36_{-0.07}^{+0.07}$ & $14.26_{-0.10}^{+0.17}$ & $14.26_{-0.10}^{+0.17}$ & $-5.13_{-0.10}^{+0.17}$ & $-0.77_{-0.20}^{+0.20}$ \\
\hline $\mathrm{C}_{\mathrm{II}}$ & $\ldots$ & $\ldots$ & $>14.76$ & $>14.79$ & $>-4.60$ & $>-1.03$ \\
\hline Al II & $\ldots$ & $13.43_{-0.19}^{+0.31}$ & $13.12_{-0.11}^{+0.07}$ & $13.12_{-0.11}^{+0.17}$ & $-6.27_{-0.11}^{+0.17}$ & $-0.72_{-0.20}^{+0.20}$ \\
\hline $\mathrm{P}_{\mathrm{II}}$ & $<13.21$ & $\ldots$ & $<12.97$ & $<12.97$ & $<-6.42$ & $<0.13$ \\
\hline CiV & $\ldots$ & $13.80_{-0.03}^{+0.04}$ & $13.58_{-0.18}^{+0.29}$ & $13.58_{-0.18}^{+0.29}$ & $-5.81_{-0.18}^{+0.29}$ & \\
\hline $\mathrm{Nv}$ & $\ldots$ & $<13.32$ & $12.85_{-0.14}^{+0.23}$ & $12.85_{-0.14}^{+0.23}$ & $-6.54_{-0.14}^{+0.23}$ & \\
\hline O VI & $\ldots$ & $14.20_{-0.02}^{+0.02}$ & $13.99_{-0.14}^{+0.36}$ & $13.99_{-0.14}^{+0.36}$ & $-5.40_{-0.14}^{+0.36}$ & \\
\hline Si IV & $\ldots$ & $13.28_{-0.02}^{+0.03}$ & $13.10_{-0.15}^{+0.25}$ & $13.10_{-0.15}^{+0.25}$ & $-6.29_{-0.15}^{+0.25}$ & \\
\hline Si III & $\ldots$ & $\ldots$ & $13.72_{-0.14}^{+0.24}$ & $13.72_{-0.14}^{+0.24}$ & $-5.67_{-0.14}^{+0.24}$ & \\
\hline
\end{tabular}

Notes.

${ }^{a}$ Comparison of measured column densities, $\log N_{a}\left(\mathrm{~cm}^{-2}\right)$, for $\mathrm{HVC}$ at $\left\langle V_{\mathrm{LSR}}\right\rangle=-133 \mathrm{~km} \mathrm{~s}^{-1}$ (integrated from $-170 \mathrm{~km} \mathrm{~s}^{-1}$ to $-100 \mathrm{~km} \mathrm{~s}^{-1}$ ).

b Abundances for neutrals and first ions $X_{i}$ are given relative to measured $\log N_{\mathrm{H}_{\mathrm{I}}}=19.39 \pm 0.01$ for the $-131 \mathrm{~km} \mathrm{~s}^{-1}$ component (Table 2). Fox et al. (2004) found $\log N(\mathrm{O} \mathrm{vI})=14.12_{-0.13}^{+0.11}$ and $\log N(\mathrm{Nv})<13.43$. Our previous Si III survey (Shull et al. 2009) measured $\log N(\mathrm{Si}$ III) $\geqslant 13.92$ as adopted here. The last column gives elemental abundances $[X / H]$ relative to solar values (Asplund et al. 2009), with ionization corrections of $0.32 \operatorname{dex}(\mathrm{S}$ II), $0.27 \mathrm{dex}$ (Si II), and $0.14 \mathrm{dex}$ (Fe II) subtracted from the first-ion abundances (Section 3.2).

the doublets of C IV $(\lambda 1548,1551), \mathrm{Nv}(\lambda 1238,1242)$, and $\operatorname{Si}$ IV $(\lambda 1394,1402)$. Neither line in the $\mathrm{N} v$ doublet $(\lambda 1238,1242)$ is seen to a $\operatorname{limit} \log N<12.99(3 \sigma)$. We use the weaker $\mathrm{N}_{\mathrm{I}} \lambda 1199.5$ line, since the other lines at $1200.2 \AA$ and $1200.7 \AA$ are blended with $V_{\mathrm{LSR}}=0$ absorption. Both Al II $\lambda 1670.78$ and Si III $\lambda 1206.50$ are mildly saturated, at equivalent widths of $\sim 215 \mathrm{~m} \AA$ and $\sim 190 \mathrm{~m} \AA$, respectively. Our inferred column densities from AOD integration of $N_{a}(v)$ accurately reflect this saturation, for line broadening of $20-30 \mathrm{~km} \mathrm{~s}^{-1}$, somewhat higher than the Doppler parameter $b=10 \mathrm{~km} \mathrm{~s}^{-1}$ inferred from $\mathrm{O}$ I and low ions (CSG03). Our previous HVC survey of Si III (Shull et al. 2009) measured $\log N \geqslant 13.60$ for this absorber, somewhat higher than the value, $\log N=$ $13.30_{-0.16}^{+0.24}$ measured here. We re-measured the $\mathrm{O}$ vi column density of this $\mathrm{HVC}$, finding fair agreement, $\log N=$ $13.58_{-0.12}^{+0.18}$ compared to $13.71 \pm 0.09$ (Fox et al. 2004) and $13.72 \pm 0.17$ (Sembach et al. 2003). We integrate between 
Table 10

PG1259+593: Summary of Column Densities and Abundances ${ }^{\mathrm{a}}$

\begin{tabular}{|c|c|c|c|c|c|c|}
\hline $\begin{array}{l}\text { Species } \\
\left(X_{i}\right)\end{array}$ & $\begin{array}{c}\text { CSG-03 } \\
\left(\log N_{X_{i}}\right)\end{array}$ & $\begin{array}{c}\text { Richter-01 } \\
\left(\log N_{X_{i}}\right)\end{array}$ & $\begin{array}{l}\text { Shull-11 } \\
\left(\log N_{X_{i}}\right)\end{array}$ & $\begin{array}{l}\text { Adopted } \\
\left(\log N_{X_{i}}\right)\end{array}$ & $\begin{array}{c}\text { Abundance }^{\mathrm{b}} \\
\log \left(N_{X_{i}} / N_{\mathrm{H}_{\mathrm{I}}}\right)\end{array}$ & $\begin{array}{c}\text { Abundance }^{\mathrm{b}} \\
{[X / H]}\end{array}$ \\
\hline O I & $15.75_{-0.24}^{+0.18}$ & $15.77_{-0.31}^{+0.37}$ & $\ldots$ & $15.85_{-0.15}^{+0.15}$ & $-4.12_{-0.15}^{+0.15}$ & $-0.81_{-0.15}^{+0.15}$ \\
\hline $\mathrm{N}_{\mathrm{I}}$ & $14.02_{-0.12}^{+0.19}$ & $13.95_{-0.21}^{+0.17}$ & $13.85_{-0.12}^{+0.12}$ & $13.85_{-0.12}^{+0.12}$ & $-6.12_{-0.12}^{+0.12}$ & $-1.95_{-0.12}^{+0.12}$ \\
\hline $\mathrm{S}_{\text {II }}$ & $14.38_{-0.11}^{+0.12}$ & $14.34_{-0.15}^{+0.12}$ & $14.35_{-0.10}^{+0.15}$ & $14.35_{-0.10}^{+0.15}$ & $-5.62_{-0.10}^{+0.15}$ & $-0.78_{-0.10}^{+0.15}$ \\
\hline Si II & $14.67_{-0.15}^{+0.20}$ & $14.56_{-0.27}^{+0.28}$ & $14.17_{-0.14}^{+0.10}$ & $14.17_{-0.14}^{+0.10}$ & $-5.80_{-0.14}^{+0.10}$ & $-1.35_{-0.14}^{+0.10}$ \\
\hline $\mathrm{Fe}$ II & $14.40_{-0.08}^{+0.17}$ & $14.16_{-0.14}^{+0.20}$ & $14.11_{-0.11}^{+0.12}$ & $14.11_{-0.11}^{+0.12}$ & $-5.86_{-0.11}^{+0.12}$ & $-1.38_{-0.11}^{+0.12}$ \\
\hline $\mathrm{C}_{\mathrm{II}}$ & $\ldots$ & $\ldots$ & $>14.45$ & $>14.45$ & $>-5.52$ & $>-1.95$ \\
\hline Al II & $13.45_{-0.16}^{+0.17}$ & $13.42_{-0.50}^{+0.30}$ & $12.89_{-0.16}^{+0.13}$ & $12.89_{-0.16}^{+0.13}$ & $-7.08_{-0.16}^{+0.13}$ & $-1.53_{-0.16}^{+0.13}$ \\
\hline $\mathrm{P}_{\mathrm{II}}$ & $<12.96$ & $<13.22$ & $<12.43$ & $<12.43$ & $<-7.54$ & $<-0.95$ \\
\hline C IV & $\ldots$ & $\ldots$ & $13.08_{-0.15}^{+0.33}$ & $13.08_{-0.15}^{+0.33}$ & $-6.89_{-0.15}^{+0.33}$ & \\
\hline $\mathrm{Nv}$ & $\ldots$ & $\ldots$ & $<12.99$ & $<12.99$ & $<-6.98$ & \\
\hline O VI & $\cdots$ & $\cdots$ & $13.58_{-0.12}^{+0.18}$ & $13.58_{-0.12}^{+0.18}$ & $-6.39_{-0.12}^{+0.18}$ & \\
\hline Si IV & $\ldots$ & $\ldots$ & $12.64_{-0.13}^{+0.17}$ & $12.64_{-0.13}^{+0.17}$ & $-7.33_{-0.13}^{+0.17}$ & \\
\hline Si III & $\ldots$ & $\ldots$ & $13.30_{-0.16}^{+0.24}$ & $13.30_{-0.16}^{+0.24}$ & $-6.67_{-0.16}^{+0.24}$ & \\
\hline
\end{tabular}

Notes.

${ }^{\text {a }}$ Comparison of measured column densities, $\log N_{a}\left(\mathrm{~cm}^{-2}\right)$, for $\mathrm{HVC}$ at $\left\langle V_{\mathrm{LSR}}\right\rangle=-128 \mathrm{~km} \mathrm{~s}^{-1}$ (integrated from -170 to $-95 \mathrm{~km} \mathrm{~s}^{-1}$ ).

${ }^{\text {b }}$ Abundances for neutrals and first ions $X_{i}$ are given relative to measured $\log N_{\mathrm{H}_{\mathrm{I}}}=19.97 \pm 0.02$ for the $\mathrm{HVC}$ components at $-126 \mathrm{~km} \mathrm{~s}^{-1}$ and $-129 \mathrm{~km} \mathrm{~s}^{-1}$ (Table 2). We adopted $\log N_{\mathrm{O}_{\mathrm{I}}}=15.85 \pm 0.15$ from Sembach et al. (2004). Fox et al. (2004) found $\log N(\mathrm{C}$ IV $)=13.26_{-0.06}^{+0.04}, \log N(\mathrm{~N} v)<12.85, \log N(\mathrm{Si}$ IV $)=12.73_{-0.03}^{+0.05}$, and $\log N(\mathrm{O}$ VI $)=13.71_{-0.09}^{+0.09}$. Our previous Si III survey (Shull et al. 2009) measured $\log N(\mathrm{Si}$ III) $\geqslant 13.60$ as adopted here. The last column gives elemental abundances $[X / H]$ relative to solar values (Asplund et al. 2009), with ionization corrections of $0.04 \mathrm{dex}$ (S II), $0.04 \mathrm{dex}$ ( $\mathrm{Si}$ II), and $0.02 \mathrm{dex}$ (Fe II) subtracted from the first-ion abundances (Section 3.2).

$V_{\mathrm{LSR}}=-170$ and $-95 \mathrm{~km} \mathrm{~s}^{-1}$, while Fox et al. (2004) used the interval from -160 to $-80 \mathrm{~km} \mathrm{~s}^{-1}$. For the $\mathrm{O}$ i column density in Complex $\mathrm{C}$, we adopt the careful measurement, $\log N_{\text {OI }}=15.85 \pm 0.15$ from Sembach et al. (2004), who fitted components as part of their study to find a deuterium ratio, $\mathrm{D} / \mathrm{H}=(2.2 \pm 0.7) \times 10^{-5}$ in this $\mathrm{HVC}$.

\section{CONCLUSIONS AND IMPLICATIONS}

The high throughput and low background of the COS allow us to observe Complex HVCs with high $\mathrm{S} / \mathrm{N}$ and improved velocity accuracy. A summary of the major issues and new observational results follows.

1. Comparison of COS/G130M and G160M data with previous HST and FUSE data shows a more complete array of transitions and elements, with higher $\mathrm{S} / \mathrm{N}$ and a betterdefined velocity range of UV absorption.

2. In general, the COS-derived column densities and abundances agree with previous UV spectroscopic studies by GHRS, STIS, and FUSE. The Complex C metallicity inferred from O I and S II lies between $10 \%$ and $30 \%$ of solar values. The better-defined velocity range of UV absorption affects some of the high-ion column densities such as O VI.

3. Using Si III as a proxy for H II, we find a substantial amount of ionized gas kinematically associated with the $\mathrm{H}_{\mathrm{I}} 21 \mathrm{~cm}$ emission, ranging from $N(\mathrm{HII}) / N(\mathrm{HI}) \approx 0.1$ (Mrk 290, PG 1259+593) to 0.8-1.5 (Mrk 817, Mrk 876). The HVCs are expected to have extended ionized atmospheres owing to photoionization, and possibly from interactions with the halo.

4. Individual clumps of Complex $C$ appear to be near virial equilibrium with pressure confinement. Their masses $\left(\geqslant 10^{5} M_{\odot}\right)$ are probably above the threshold for survival against dissipation by interface instabilities.

5. High-ionization states ( $\mathrm{C}$ IV, Nv, Si IV, O vi) are seen in all four sight lines, with column densities ranging over factors of three to five and ratios consistent with multiphase ionization processes: $N(\mathrm{Si}$ IV $) / N(\mathrm{O}$ vI $) \approx 0.05-0.11$, $N(\mathrm{C}$ IV $) / N(\mathrm{O}$ vI $) \approx 0.3-0.5, N(\mathrm{~N} v) / N(\mathrm{O}$ vI $) \approx 0.07-0.13$, and $N(\mathrm{Si}$ IV $) / N(\mathrm{Si}$ III $) \approx 0.1-0.2$. The observed ratios Si IV/ $\mathrm{C}_{\mathrm{IV}} \approx 0.15-0.36$ are inconsistent with photoionization models for the low ions.

6. Models of high-ion production and C IV, Nv, O vi line ratios in Galactic halo gas (Indebetouw \& Shull 2004a, 2004b; Gnat \& Sternberg 2007) suggest radiative cooling and conductive heating. They are inconsistent with CIE, which produces more $\mathrm{NV}$ and less $\mathrm{O}$ vi than observed: $N(\mathrm{~N} \mathrm{v}) / N(\mathrm{O}$ vI $) \approx 0.3$ and $N(\mathrm{C}$ IV $) / N(\mathrm{O}$ vI $) \approx 0.1$. The low $\mathrm{Nv}$ probably reflects the nitrogen underabundance observed in the neutral components of many HVCs.

As noted in the introduction, Complex $\mathrm{C}$ is a large $(M \sim$ $10^{7} M_{\odot}$ ) HVC with substantial amounts of neutral and ionized gas, falling toward the Galactic plane with an average mass flow of $0.1 M_{\odot} \mathrm{yr}^{-1}$ of low-metallicity gas (10\%-30\% solar). Over the entire Galactic halo, as probed by Si III (Shull et al. 2009), HVCs may provide sufficient gaseous infall, $\sim 1 M_{\odot} \mathrm{yr}^{-1}$ to help replenish some of the $2-4 M_{\odot} \mathrm{yr}^{-1}$ of star formation in the disk. Future HVC studies with COS will include sight lines passing through Complex M (Danly et al. 1993; Yao et al. 2011), Complex A (van Woerden et al. 1999), and the Smith Cloud (Lockman et al. 2008). With more accurate metal-ion abundances and ionization corrections, we may soon be able to assess the relative abundance ratios of different elements $(\mathrm{S}$, $\mathrm{Si}, \mathrm{O}, \mathrm{Fe}, \mathrm{N}$, and perhaps $\mathrm{P}$ and $\mathrm{Al}$ ) seeking to find non-solar 
ratios indicative of chemical history. Nitrogen is underabundant relative to $\mathrm{C}$ and $\mathrm{S}$, which suggests that Complex $\mathrm{C}$ gas is chemically young.

The abundances of refractory elements $(\mathrm{Si}, \mathrm{Al}, \mathrm{Fe})$ relative to undepleted $\mathrm{S}$ could limit the amount of dust, which is expected to be small, given the lack of clear evidence for infrared emission from Complex $\mathrm{C}$. Typical values for these four sight lines are $(\mathrm{S} / \mathrm{Fe}) \approx 1.0-1.7,(\mathrm{Si} / \mathrm{Fe}) \approx 1.2-1.7$, and $(\mathrm{Al} / \mathrm{Fe})$ $\approx 0.06-0.10$. Because this range is within the uncertainty of the measurements, it is difficult to draw any firm conclusions. The $[\alpha / \mathrm{Fe}]$ ratios are interpreted as the ratio of massive-star nucleosynthesis (and core-collapse $\mathrm{SNe}$ ) to nucleosynthesis from older stars (and Type Ia SNe, which produce more Fe).

It is our pleasure to acknowledge the thousands of people who made HST Servicing Mission 4 a huge success. We thank Brian Keeney, Stéphane Béland, and the rest of the COS/GTO team for their work on the calibration and verification of the early COS data. We thank Andrew Fox for helpful discussions of his previous studies of the high ions toward Complex $\mathrm{C}$ and Mark Giroux for insights on photoionization modeling of HVCs. We also appreciate comments from the referee, Kenneth Sembach, on physical conditions in HVCs and details of the COS data analysis. This work was supported by NASA grants NNX08AC146 and NAS5-98043 and the Astrophysical Theory Program (NNX07-AG77G from NASA and AST07-07474 from NSF) at the University of Colorado at Boulder.

\section{REFERENCES}

Asplund, M., Grevesse, N., Sauval, A. J., \& Scott, P. 2009, ARA\&A, 47, 481 Blagrave, K., Lockman, F. J., \& Martin, P. G. 2010, in ASP Conf. Ser., The Dynamic ISM: A Celebration of the Canadian Galactic Plane Survey, ed. R. Kothes, T. L. Landecker, \& A. S. Willis (San Francisco, CA: ASP), 156 Blitz, L., \& Robishaw, T. 2000, ApJ, 541, 675

Collins, J. A., Shull, J. M., \& Giroux, M. L. 2003, ApJ, 585, 336 Collins, J. A., Shull, J. M., \& Giroux, M. L. 2004, ApJ, 605, 216 Collins, J. A., Shull, J. M., \& Giroux, M. L. 2005, ApJ, 623, 196 Collins, J. A., Shull, J. M., \& Giroux, M. L. 2007, ApJ, 657, 271 Collins, J. A., Shull, J. M., \& Giroux, M. L. 2009, ApJ, 705, 962 Danforth, C. W., Keeney, B. A., Stocke, J. T., Shull, J. M., \& Yao, Y. 2010, ApJ, 720,976

Danly, L., Albert, E., \& Kuntz, K. D. 1993, ApJ, 416, L29

Dekel, A., \& Birnboim, Y. 2006, MNRAS, 368, 2

Diehl, R., Halloin, H., Kretschmer, K., et al. 2006, Nature, 439, 45

Erb, D. K., Shapley, A. E., Pettini, M., et al. 2006, ApJ, 644, 813

Fox, A. J., Savage, B. D., \& Wakker, B. P. 2006, ApJS, 165, 229
Fox, A. J., Savage, B. D., Wakker, B. P., et al. 2004, ApJ, 602, 738

Fox, A. J., Wakker, B. P., Savage, B. D., et al. 2005, ApJ, 630, 332

Gibson, B. K., Giroux, M. L., Penton, S. V., et al. 2000, AJ, 120, 1830

Gibson, B. K., Giroux, M. L., Penton, S. V., et al. 2001, AJ, 122, 3280

Gilmore, G. 2001, in ASP Conf. Ser. 230, Galaxy Disks \& Disk Galaxies, ed. J. G. Funes \& E. M. Corsini (San Francisco, CA: ASP), 3

Gnat, O., \& Sternberg, A. 2007, ApJS, 168, 213

Green, J., et al. 2011, ApJ, submitted

Heitsch, F., \& Putman, M. 2009, ApJ, 698, 1485

Indebetouw, R., \& Shull, J. M. 2004a, ApJ, 605, 215

Indebetouw, R., \& Shull, J. M. 2004b, ApJ, 607, 309

Kahn, F. D., \& Woltjer, L. 1959, ApJ, 130, 705

Kalberla, P. M. W., Burton, W. B., Hartmann, D., et al. 2005, A\&A, 440, 775

Kereš, D., Katz, N., Fardal, M., Davé, R., \& Weinberg, D. H. 2009, MNRAS, 395,160

Kriss, G. A. 2011, Improved Medium Resolution Line Spread Functions for COS FUV Spectra (COS ISR2011-01; Baltimore: STScI)

Kwak, K., \& Shelton, R. L. 2010, ApJ, 719, 523

Lockman, F. J., Benjamin, R. A., Heroux, A. J., \& Langston, G. J. 2008, ApJ, 679, L21

Lockman, F. J., Murphy, E. M., Petty-Powell, S., \& Urick, V. 2002, ApJS, 140, 331

Moore, B., \& Davis, M. 1994, MNRAS, 270, 209

Morton, D. C. 2003, ApJS, 149, 205

Osterman, S., Green, J., Froning, C., et al. 2011, Ap\&SS

Pagel, B. E. J. 1994, in The Formation and Evolution of Galaxies, ed. C. MunezTunón \& F. Sanchez (Cambridge: Cambridge Univ. Press), 149

Reynolds, R. J. 1991, ApJ, 372, L17

Richter, P., Sembach, K. R., Wakker, B. P., et al. 2001, ApJ, 559, 318

Robitaille, T. P., \& Whitney, B. A. 2010, ApJ, 710, L17

Sembach, K. R., \& Savage, B. D. 1992, ApJS, 83, 147

Sembach, K. R., Savage, B. D., Lu, L., \& Murphy, E. M. 1999, ApJ, 515, 108

Sembach, K. R., Wakker, B. P., Savage, B. D., et al. 2003, ApJS, 146, 165

Sembach, K. R., Wakker, B. P., Tripp, T. M., et al. 2004, ApJS, 150, 387

Shull, J. M., Jones, J. R., Danforth, C. W., \& Collins, J. A. 2009, ApJ, 699, 754

Slavin, J. D., Shull, J. M., \& Begelman, M. C. 1993, ApJ, 407, 83

Spitzer, L. 1956, ApJ, 124, 20

Spitzer, L. 1978, Physical Processes in the Interstellar Medium (New York: Wiley)

Thom, C., Peek, J. E. G., Putman, M. E., et al. 2008, ApJ, 684, 364

Tremonti, C. A., Heckman, T. M., Kauffmann, G., et al. 2004, ApJ, 613, 898

Tufte, S. L., Reynolds, R. J., \& Haffner, L. M. 1998, ApJ, 504, 773

van Woerden, H., Schwarz, U. J., Peltier, R. F., Wakker, B. P., \& Kalberla, P. M. W. 1999, Nature, 400, 138

Wakker, B. P. 2001, ApJS, 136, 463

Wakker, B. P., Howk, J. C., Savage, B. D., et al. 1999, Nature, 402, 388

Wakker, B. P., Kalberla, P. M. W., van Woerden, H., et al. 2001, ApJS, 136, 537

Wakker, B. P., Lockman, F. J., \& Brown, J. M. 2011, ApJ, 728, 159

Wakker, B. P., Savage, B. P., Sembach, K. R., et al. 2003, ApJS, 146, 1

Wakker, B. P., \& van Woerden, H. 1997, ARA\&A, 35, 217

Wakker, B. P., York, D. G., Howk, J. C., et al. 2007, ApJ, 670, L113

Winter, L. M., Danforth, C., Vasudevan, R., et al. 2011, ApJ, 728, 28

Yao, Y., Shull, J. M., \& Danforth, C. W. 2011, ApJ, 728, L16 